\title{
Probabilistic Shaping for the Optical Phase Conjugation Channel
}

Yankov, Metodi Plamenov; Hansen, Henrik Enggaard; Da Ros, Francesco; Kaminski, Pawel Marcin; Porto da Silva, Edson; Galili, Michael; Oxenlowe, L.K.; Forchhammer, Søren

\section{Published in:}

IEEE Journal of Selected Topics in Quantum Electronics

Link to article, DOI:

10.1109/JSTQE.2020.3024843

Publication date:

2021

Document Version

Peer reviewed version

Link back to DTU Orbit

Citation (APA):

Yankov, M. P., Hansen, H. E., Da Ros, F., Kaminski, P. M., Porto da Silva, E., Galili, M., Oxenlowe, L. K., \& Forchhammer, S. (2021). Probabilistic Shaping for the Optical Phase Conjugation Channel. IEEE Journal of Selected Topics in Quantum Electronics, 27(2), [7700716 ]. https://doi.org/10.1109/JSTQE.2020.3024843

\section{General rights}

Copyright and moral rights for the publications made accessible in the public portal are retained by the authors and/or other copyright owners and it is a condition of accessing publications that users recognise and abide by the legal requirements associated with these rights.

- Users may download and print one copy of any publication from the public portal for the purpose of private study or research.

- You may not further distribute the material or use it for any profit-making activity or commercial gain

- You may freely distribute the URL identifying the publication in the public portal 


\title{
Probabilistic Shaping for the Optical Phase Conjugation Channel
}

\author{
Metodi P. Yankov, Member, IEEE, Henrik E. Hansen, Francesco Da Ros, Senior Member, OSA, Member, IEEE, \\ Pawel M. Kaminski, Edson Porto da Silva, Member, OSA, Senior Member, IEEE, \\ Michael Galili, Member, OSA,Member, IEEE, Leif K. Oxenløwe, Fellow, OSA,Member, IEEE, \\ and Søren Forchhammer, Member, IEEE,
}

\begin{abstract}
Probabilistic constellation shaping is studied and developed for optical phase conjugation (OPC)-based nonlinearity compensation of Kerr nonlinearities in optical fiber links. The mid-link OPC scenario is considered for dispersion compensated systems. It is demonstrated in simulations and experimentally that transmission strategies optimal for classical additive white Gaussian noise (AWGN) channels can be sub-optimal for these systems without nonlinearity compensation. On the contrary, when nonlinearity compensation is applied with mid-link OPC, the channel noise is demonstrated to be Gaussian and AWGNlike transmission strategies thus remain effective. A channelagnostic probability mass function (PMF) optimization algorithm is proposed for the input constellation in order to further improve the shaping gains in both scenarios. Operating arbitrary PMFs on arbitrary channels is enabled by a channel-agnostic digital signal processing (DSP) chain. After $\approx 2000 \mathrm{~km}$ of transmission, mid-link OPC is demonstrated to provide $\approx 1 \mathrm{~dB}$ of gain in effective SNR, which translates to $\approx 0.4$ bits/QAM symbol of gain in achievable information rate. The gain is then increased by an extra $\approx 0.2$ bits/QAM symbol by applying probabilistic shaping.
\end{abstract}

Index Terms-Constellation shaping, OPC, DSP, optical communications, nonlinearities

\section{INTRODUCTION}

$\mathbf{K}$ ERR nonlinearities are the key effect currently preventing optical fiber systems from operating at the high signal to noise ratio (SNR) regime and high data rates, respectively. Particularly in wavelength division multiplexed (WDM) systems, cross-phase modulation (XPM) and self phase modulation (SPM) result in severe nonlinear interference and limit the performance of standard digital transceivers. Nonlinearities have been the subject of a broad range of theoretical and experimental studies [1], [2], [3], [4], [5], with no one clear and practical solution coming close to unlocking a significant portion of the fiber nonlinear regime for data transmission. Several digital techniques deserve mentioning in this category:

1) Theoretically, the nonlinear frequency division multiplexed (NFDM) systems have been studied as a replacement for WDM systems. For lossless fibers, the former sup-

Metodi P. Yankov, Henrik E. Hansen, Pawel M. Kaminski, Michael Galili, Leif K. Oxenløwe and Søren Forchhammer are with the Department of Photonics Engineering, Technical University of Denmark (DTU), Oersteds Plads 343, 2800 Kgs. Lyngby, Denmark e-mail: meya@fotonik.dtu.dk.

Edson Porto da Silva was with the Department of Photonics Engineering, DTU, and is now with the Center of Electrical Engineering and Informatics (CEEI), Electrical Engineering Department (DEE), Federal University of Campina Grande (UFCG), Brazil.

Manuscript received XXX XX, XXXX; revised XXX XX, XXXX port undistorted pulse propagation and thus improved performance using the widespread and standard single mode fibers (SSMFs). While some experimental studies have been performed on NFDM [6], [7], practical transceiver penalties and the above-mentioned practical fiber loss currently limit the applicability of NFDM.

2) Digital signal pre- and post-processing [3], [8], [9], [10] of nonlinearities allow for some of the SPM and XPM to be compensated for. Typically, however, signals are subjected to propagation in a non-static network with random and ever-changing interference patterns. Furthermore, digital signal processing (DSP) is inherently limited to within the receiver/transmitter bandwidth. These two factors constrain the achievable SNR gains through DSP of fiber nonlinearities to $\approx$ $1 \mathrm{~dB}$ [11] at the cost of significant computational complexity, related to significant power consumption penalties.

3) Constellation shaping (CS) is a generic method for the maximization of achievable information rates (AIRs) of noisy channels [12], [13], [14]. Shaping aims at modifying the statistics of the signal waveform to better match the channel conditions and at the same time support high data rates. Two major classes of CS can be defined - probabilistic shaping (PS) and geometric shaping (GS). Assume a typical quadrature amplitude modulation (QAM) constellation is employed for digital transmission of data. The former class aims at modifying the signal statistics by optimizing the probability of occurrence of signaling points from the constellation. The latter aims at optimizing the location of the points in the I/Q plane. Theoretically, joint optimization is required to maximize the AIR and achieve the capacity of the channel. While effective shaping strategies in terms of reducing the gap to the Shannon limit [15] can be devised in the linear region of transmission [16], [17], [18], [19], CS for the nonlinear region is up to date theoretically (in terms of estimating theoretical limits of shaping gains) and practically (in terms of devising transceivers to achieve the gains) an open problem, with most solutions beneficial up to the weakly nonlinear region [20], [21], [22], [23]. Typically, the problem is approached using the mismatched decoding principle (to be detailed later in the paper) and optimization for mismatched channels. Some general capacity bounds have been derived for basic communication channels with a mismatched receiver, e.g. [24], [25] and references therein. Relatively recently has practical optimization using mismatched decoding principles been applied to the nonlinear optical channel [26], [22], [27], 
[28], [29].

Optical signal processing-based nonlinearity compensation through optical phase conjugation (OPC) [30] has several significant advantages in light of above. In the simplest of terms, OPC compensates for nonlinear distortions, accumulated in some sections of the link by accumulating nonlinearities of opposite sign and equal magnitude via propagation in different sections of the link. The simplest example is the mid-link OPC, which employs four-wave mixing (FWM) in a highly nonlinear medium at the middle of the transmission link to produce a conjugated copy of the signal replacing the original for further propagation. Exploiting the Hermitian symmetry of the nonlinear Schrödinger equation (NLSE) governing signal propagation through the optical fiber, the conjugated copy in the second part of the link accumulates the exact opposite nonlinearities to the first half, which then cancel. The process is entirely optical, thus the limited bandwidth constraint and high power consumption of DSP can potentially be efficiently overcome.

Ideal compensation with OPC inherently requires power and dispersion symmetry in the propagating paths before and after conjugation, which is not always possible in practice, especially with lumped amplification links unless specific link design strategies are employed [31], [32]. Nonstatic copropagating interference patterns in dynamic network scenarios pose another challenge regarding symmetry. While some improvements can be achieved in such cases [33], the symmetry generally remains a limitation to the expected gains. Practical ways to address these issues are e.g. 1) to increase the granularity of conjugation stages, which has shown promising results [34], [35]; 2) using distributed Raman amplification to increase the power symmetry [36], [37]; or 3) designing dispersion-optimized transmission links [31].

For the rest of the paper, the standard mid-link OPC scenario for legacy transmission links based on lumped EDFA amplification is discussed. Partial symmetry will then be mainly ensured by the dispersion-compensated transmission.

Combination of OPC with other nonlinearity compensation techniques, e.g. digital back-propagation in [38] and Volterrabased equalization in [39], [40], has been recently explored, showing that the digital and optical techniques can supplement each other. Ultimately, they aim at compensation of the same effects and are thus not complimentary. The main contribution of this paper is to study experimentally and through simulations PS as a complimentary technique to OPC, in particular, to mid-link OPC.

This paper extends our previous contributions on the combination of PS with OPC [26], [41] by describing the theoretical foundations of constellation shaping and proposing a more robust and universal channel-agnostic optimization algorithm. Universality stems from the fact that the algorithm is independent of the actual channel law and the rest of the processing steps between the input PMF and the cost function, in this case - the AIR. This paper also extends the experimental demonstration in [41] with deeper analysis of the optimal constellation shapes and a detailed description of the DSP functions, necessary to achieve shaping gains in non-standard channels, such as the mid-link OPC. Similar to

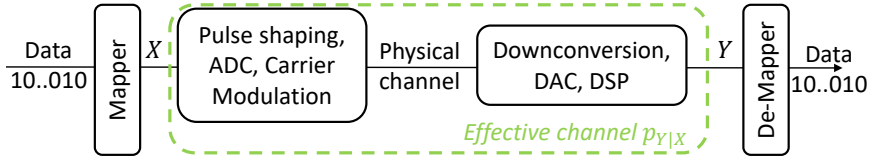

Fig. 1. Basic communication system model.

the optimization algorithm designed in this work, the DSP chain is universal and channel-agnostic. Flexibility is a key requirement to achieve efficiency, which will be increasingly important for future optical networks. Accordingly, universality will be a key requirement for the transceivers to achieve flexibility. This work can thus be extended to other standard and non-standard scenarios alike.

The rest of the paper is organized as follows. In Section II, the foundations of constellation shaping for digitally modulated communication systems are briefly reviewed. In Section III, the mid-link OPC channel is presented. A universal DSP chain is presented in Section IV. Simulation-based optimization and analysis of optimal constellation shapes under practical constraints is presented in Section V. Experimental validation is performed and detailed in Section VI. Further discussion and future work is offered in Section VII, and conclusion in Section VIII.

\section{INFORMATION THEORY BACKGROUND}

\section{A. Notation}

Throughout the paper, the following notation shall be used. Random variables are denoted with capital letters, e.g. $X$. Realizations of variables with small letters, e.g. $x$ for scalars and $\mathbf{x}$ for vectors, $x_{k}$ being the $k$-th element in a sequence of outcomes $x_{1}^{K}$ of length $K$. Similarly, a sequence of random variables is denoted $X_{1}^{K}$. The probability of the outcome $x$ is denoted $p(x)$, the probability mass function of the discrete variable $X$ is denoted $P_{X}$, whereas for continuous variables, the probability density function is denoted $p_{X}$. Other notations are defined on occurrence.

\section{B. Entropy, mutual information and capacity}

A standard communication system is assumed, where data are to be sent from a transmitter to a receiver over a noisy channel, see Fig. 1. Data bits are mapped to constellation symbols $X$, which then modulate (after pulse shaping and digitalto-analog conversion (DAC)) the carrier wave. The modulated carrier propagates through a physical channel, which is generally noisy and subject to memory and interference. At the receiver, analog-to-digital conversion is performed on the baseband signal, followed by a DSP chain. The purpose of the DSP is to pre-process the signal so that simpler detection of the received symbols $Y$ can be performed. The effective channel as seen by the demapper can be represented by the PDF $p_{Y \mid X}$. 
The maximum rate at which data can be sent through the channel in Fig. 1 with asymptotically vanishing probability of error is given by the Shannon capacity $\mathcal{C}$ [15]

$$
\begin{aligned}
\mathcal{C} & =\max _{P_{X}} \mathcal{I}(X ; Y) \\
& =\max _{P_{X}} \mathcal{H}(X)-\mathcal{H}(X \mid Y), \text { bits/channel use, }
\end{aligned}
$$

where $X$ and $Y$ are the input and output of the channel in one channel use, respectively, $\mathcal{I}(X ; Y)$ is the mutual information (MI) between $X$ and $Y, \mathcal{H}(X)$ is the entropy of $X$ and $\mathcal{H}(X \mid Y)$ is the conditional entropy of $X$ when $Y$ has been observed. Constrained capacity can also be defined by subjecting the optimization to practical constraints. An example of such constraints are average power (related to energy consumption restrictions) $\mathbb{E}\left[|X|^{2}\right] \leq P_{a v}$, or peak amplitude $\max _{x \in \mathcal{X}}|x|<A$ (related to physical component limitations, such as nonlinear amplifiers and modulators). Channel uses are drawn from the degrees of freedom available for modulating an electromagnetic wave, namely phase, bandwidth occupancy, symbol duration, and space orientation. In optical communications, the space dimension describes the polarization of light, as well as the modes of propagation, supported by the waveguide/fiber. In this work, space is restricted to the two polarizations of light, i.e. dual polarization single mode transmission is assumed. Depending on the definition, a channel use can span a multitude of the available dimensions. For example, a channel use can be defined as a single time slot on a single carrier frequency and single polarization of the electromagnetic wave, as well as a whole frame of information. The variables $X$ and $Y$ in the first case are scalars, whereas in the latter case are vectors. For the rest of the paper, one channel use will be defined as a symbol period for both I and $\mathrm{Q}$ dimensions of the complex-valued representation of the wave. Without loss of generality, complex-valued QAM constellations are assumed for $\mathcal{X}$, i.e. a channel use is defined as a QAM symbol. The efficiency and MI are then measured in bits/QAM symbol. Multiplying the per-channel-use MI by the frequency of modulation and the number of spatial dimensions utilized by the system thus results in the total data rate in bits/s carried by that optical carrier.

Digital communication systems are assumed, i.e., $X$ is discrete. Practical constraints (e.g. finite frame lengths and dynamic ranges of electronics) also dictate $X$ to live in an alphabet $\mathcal{X}$ of finite size $|\mathcal{X}|$. The simplest example is the on-off keying modulation format, for which the alphabet of $X$ is $X \in \mathcal{X}=\{0,1\}$. The maximization in (1) is over all possible PMFs of the input $X$. The entropy $\mathcal{H}(X)$ describes what the information content of the variable $X$ is, and represents an upper limit to the information rate. It is defined as $\mathcal{H}(X)=-\sum_{x \in \mathcal{X}} p(x) \log _{2} p(x)$. The entropy, and thus the information rate is ultimately bounded by the size of the communication alphabet to $\mathcal{H}(X) \leq \log _{2}|\mathcal{X}|$, with equality achieved iff the PMF is uniform. Increasing the ultimate limit on the information rate thus dictates that larger alphabets are used in order to improve the efficiency of the channel uses. The conditional entropy instead describes how much the uncertainty about $X$ is reduced by observing $Y$. Increasing the channel use efficiency through alphabet expan- sion and increasing $\mathcal{H}(X)$ typically is related also to increased $\mathcal{H}(X \mid Y)$, resulting in the ultimate trade-off highlighted in (1) between richness of the information the transmitter is trying to convey and the corresponding sensitivity of this information to detrimental channel effects.

The relations above are best described by the following simple examples:

1) In an ideal channel, $Y$ is a deterministic, bijective function of $X$. Observing $Y$ thus tells us exactly the information content of $X, \mathcal{H}(X \mid Y)=0$ and $\mathcal{I}(X ; Y)=$ $\mathcal{H}(X)$, i.e., the information rate is maximized.

2) In a completely noisy channel, the message $X$ is altered so much during transmission that $Y$ and $X$ are statistically independent, $\mathcal{H}(X \mid Y)=\mathcal{H}(X)$ and the error-free information rate is $\mathcal{I}(X ; Y)=0$.

3) In a practically relevant scenario, some information is lost during transmission, but $\mathcal{H}(X)>\mathcal{H}(X \mid Y)>0$ and $\mathcal{I}(X ; Y)>0$.

In a channel use-efficient scenario, $\mathcal{H}(X)>1$ and $\mathcal{I}(X ; Y)>$ 1. It is critical to note that constrained maximization of $\mathcal{H}(X)$ generally does not yield maximization of $\mathcal{I}(X ; Y)$. The optimum input distribution is thus generally non-uniform and subject to the optimization of (1).

Communication systems nowadays are pushed to be as transparent and as universal as possible w.r.t. the vast amount of different information sources that are to be processed. In order to achieve that, a uniform distribution on the data is assumed, and it is assumed that data are presented as bits. The process of encoding the uniformly distributed binary user data into a signal $X$ that has some optimized PMF is referred to as distribution matching (DM). It has been demonstrated in a variety of studies that the symbol-wise MI gains achieved by PS can mostly be harvested with binary data using various DM techniques [16], [18], [19], including in optical fiber communication scenarios [23], [22], [29]. Distribution matching is thus out of the scope of this paper. Instead, attention is given to optimization of the PMF, also known as probabilistic shaping.

\section{Achievable information rates}

The MI in (1) can be estimated from sequences of channel uses as

$$
\begin{aligned}
\mathcal{I}(X ; Y) & =\lim _{K \rightarrow \infty} \frac{1}{K} \mathcal{I}\left(X_{1}^{K} ; Y_{1}^{K}\right) \\
& =\lim _{K \rightarrow \infty} \frac{1}{K}\left(\mathcal{H}\left(X_{1}^{K}\right)-\mathcal{H}\left(X_{1}^{K} \mid Y_{1}^{K}\right)\right) .
\end{aligned}
$$

Assuming stationarity of the PMF (PDF in the continuous case), the entropy can be estimated from a sequence of outcomes as [42]

$$
\mathcal{H}\left(X_{1}^{K}\right)=-\lim _{K \rightarrow \infty} \log _{2} p\left(x_{1}^{K}\right)=-\lim _{K \rightarrow \infty} \sum_{k=1}^{K} \log _{2} p\left(x_{k}\right)
$$

where the last equality follows from the assumption (typical in digital communications) that the constellation symbols similarly to the data are independent, identically distributed (i.i.d.) and the joint distribution $p\left(x_{1}^{K}\right)$ factorizes into the 
product of its marginals $\prod_{1}^{K} p\left(x_{k}\right)$. Should this assumption fail, the last equality is replaced by an inequality $(\leq)$. In fact, replacing the joint distribution $P_{X_{1}^{K}}$ by any other valid distribution $Q_{X_{1}^{K}}$ leads to an upper bound on the entropy [42]

$$
\mathcal{H}\left(X_{1}^{K}\right) \leq \lim _{K \rightarrow \infty}-\log _{2} q\left(x_{1}^{K}\right),
$$

which is known as the mismatched decoding principle, and is further exemplified below.

The independence assumption in (3) is in practice, and especially in the optical fiber channel too strong for the distribution $P_{X \mid Y}$. In the latter case, memory is introduced due to the interaction of fiber nonlinearities, dispersion and noise, which cannot be completely removed by the DSP chain. Detectors for channels with memory are typically based on the maximum-likelihood sequence estimation [43], [44], [45], [46], whose complexity grows exponentially with both the memory and the alphabet cardinality (and thus the target channel use efficiency). This constrains standard, fast optical receivers to memoryless processing, effectively applying the mismatched decoding principle in the following way. The conditional PMF $P_{X_{1}^{K} \mid Y_{1}^{K}}$ is replaced by an auxiliary $P M F$ $Q_{X_{1}^{K} \mid Y_{1}^{K}}$, defined as

$$
Q_{X_{1}^{K} \mid Y_{1}^{K}}=\frac{P_{X_{1}^{K}} \cdot q_{Y_{1}^{K} \mid X_{1}^{K}}}{\sum_{X_{1}^{K}} P_{X_{1}^{K}} \cdot q_{Y_{1}^{K} \mid X_{1}^{K}}},
$$

where the auxiliary function $q_{Y_{1}^{K} \mid X_{1}^{K}}$ is also referred to as an auxiliary channel. As mentioned, applying the mismatched decoding principle results in upper-bounding the entropy $\mathcal{H}(X \mid Y)$, and thus lower-bounding the MI $\mathcal{I}(X ; Y)$. A detector applying the auxiliary channel function to estimate the transmitted symbols will thus at most achieve a rate, which is a lower bound on the MI [42], [24]. This rate is routinely referred to as the information rate, or AIR of the system. Consequently, the optimization in (1) can be performed for the AIR instead of the MI, resulting in an optimized AIR [24], but generally not in capacity. Nevertheless, AIR optimization has been successfully performed to boost the throughput of optical fiber systems [23], [22], [47], [48], [29]. It is also the figure of merit used in this work.

\section{Gaussian channel example}

One of the most common channel models is the additive white Gaussian noise (AWGN) model, for which

$$
\begin{gathered}
Y=X+N \\
p_{Y \mid X}(y, x)=p_{N}(y-x)=\mathcal{N}\left(x, \sigma_{N}^{2}\right),
\end{gathered}
$$

where $\mathcal{N}\left(\mu, \sigma^{2}\right)$ is the Gaussian PDF with mean $\mu$ and variance $\sigma^{2}$. This channel is memoryless and the optimal detector function $P_{X \mid Y}$ is thus straight-forward to apply. For such channels, the optimal discrete PMF $P_{X}$ in the limit of $|\mathcal{X}| \rightarrow \infty$ is given by the Maxwell-Boltzmann (MB) distribution [49], defined as

$$
p(x)=\frac{\exp \left(-\lambda|a x|^{2}\right)}{\sum_{x \in \mathcal{X}} \exp \left(-\lambda|a x|^{2}\right)},
$$

where $\lambda$ is a scaling factor that parametrizes the distribution and $a$ is a scaling factor ensuring a fixed average power $P_{a v}=$ $\sum_{x \in \mathcal{X}} p(x)|a x|^{2}$. In the general case of alphabets of practical sizes, this PMF maximizes entropy for a given average energy [50], but is generally not the theoretical optimum in the MI sense. Algorithms for finding the constellation-constrained optimum were proposed in [51] for scalar AWGN and [52] for multiple-input multiple-output AWGN channels, and are variants of the classical Blahut-Arimoto algorithm (BAA) for discrete, memoryless channels [53]. The MB approximation (8) remains attractive in practical cases because it imposes a functional form on the PMF and can still be applied to obtain near-optimum results [49], [50] for the AWGN channel, reducing the optimization to a simple 1-D line search for the optimum parameter $\lambda$.

For special channels, this reduction may prove to be too coarse, resulting in a penalty. Variants of the BAA can be applied in such cases without guarantee of optimality or convergence. Examples include the experimental optimization of a mid-link OPC-based channel [26] and the simulation-based optimization for channels with memory [54] later applied to the optical fiber [27].

In Fig. 2, an example optimum MB PMF for a Gaussian channel with an $\mathrm{SNR}=15 \mathrm{~dB}$ and a 64QAM constellation set $\mathcal{X}$ is given as a histogram of the received signal $Y$, together with the histogram of the received signal of uniform input PMF. For an AWGN channel, the optimum parameter $\lambda$ is positive, making points with high amplitude to appear with lower probability. For a fixed average energy, this results in a scaling of the constellation, such that the Euclidean distance between constellation points is increased. Even-though the entropy $\mathcal{H}(X)$ is reduced, this distance results in an improved detectability of the points (represented in a lower $\mathcal{H}(X \mid Y)$ ), and overall improved $\mathcal{I}(X ; Y)$. In this case, the receiver applies the function $Q_{X \mid Y}=P_{X \mid Y}$, the AIR thus corresponds to the MI, and optimization of the AIR thus corresponds to obtaining the constellation constrained capacity (CCC) of the channel (constrained to the symbol alphabet $\mathcal{X}$ ). The CCC should not be confused with the capacity of the channel, which implies optimization over all alphabets and input PDFs, including continuous ${ }^{1}$. For an AWGN channel, analytical powerconstrained optimization over continuous PDFs is possible, resulting in a Gaussian PDF optimum [53].

\section{E. Standard optical fiber channel example}

Signal propagation through the optical fiber is subjected most importantly to chromatic dispersion (CD), attenuation and the Kerr nonlinearity. To combat attenuation, periodic amplification through an erbium doped fiber amplification (EDFA) is typically applied in fiber systems. Amplification in an EDFA is inevitably accompanied by amplified spontaneous emission (ASE), which manifests itself in the form of an AWGN. Chromatic dispersion is well-modeled by an all-pass filter and is typically compensated at the receiver side by a time or frequency domain filter [55]. Loss and CD by themselves are trivial to compensate with these methods in modern

\footnotetext{
${ }^{1}$ Similarly, constellation-size constrained capacity can be defined, which implies joint probabilistic and geometric shaping for an alphabet of given size.
} 

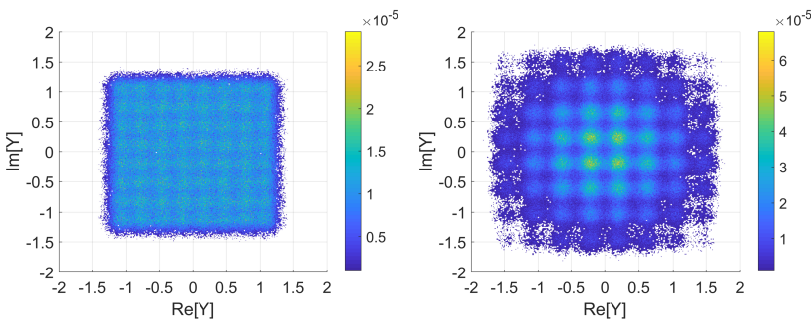

Fig. 2. Histogram of the received signal $Y$ in the case of an AWGN channel with SNR=15 dB. Left: uniform input PMF, $\mathcal{H}(X)=$ 6 bits/QAM symbol, $\mathcal{I}(X ; Y)=4.68$ bits/QAM symbol; Right: the optimal MB PMF, $\mathcal{H}(X)=5.55$ bits/QAM symbol, $\mathcal{I}(X ; Y)=$ 4.91 bits/QAM symbol. Identical scale in both cases, exemplifying the increased $\mathrm{ED}$ in the MB case.

systems. However, their interplay with the nonlinear effects, especially in WDM systems where XPM is present is currently the main limitation to the AIRs of fiber communication links. Furthermore, the effective SNR

$$
S N R_{e f f}=\frac{\mathbb{E}\left[|X|^{2}\right]}{\mathbb{E}\left[|Y-X|^{2}\right]}
$$

as seen by the receiver depends on the input distribution through the nonlinearities, invalidating all necessary assumptions for the optimality of the BAA and the near-optimality of the MB-based optimization. Combating nonlinearity is the main subject of this work. Details about this channel will be introduced and discuss later in the paper. At this stage, the MI of a standard point-to-point fiber link is compared to the corresponding MI of a trivial case where the nonlinearity is switched off. An illustrative example is considered for the performance of the central channel of a five WDM channel signal with dual polarization 64QAM 16 GBd symbol rate and $25 \mathrm{GHz}$ channel spacing, pulse shaped by a square-root raised cosine filter with a roll-off of 0.01 . The signal is propagated through 20x100 km spans of SSMF, amplified by an EDFA with a noise figure (NF) of $5 \mathrm{~dB}$ at each span. After CD compensation, matched filtering and downsampling, a Gaussian auxiliary channel is applied in all cases to estimate the AIR through the MI as discussed above. Optimizations through the MB approximation mentioned above and a variant of the BAA adopted to the optical fiber [22], [26] are compared. The results are given in Fig. 3 as a function of the launch power per channel.

In the case where the nonlinearity is switched off, the channel collapses to a pure AWGN channel, and as mentioned, the BA and the MB-based optimizations converge to the CCC. As the power increases, $S N R_{e f f}$ increases correspondingly and the AIR eventually approaches the upper bound imposed by the limited size of the constellation of $\max \mathcal{I}(X ; Y)=$ $\max \mathcal{H}(X)=\log _{2}|\mathcal{X}|=6$ bits/symbol for the 64QAM considered here. In the case of fiber propagation, when increasing the launch power, the nonlinear effects become more and more pronounced and ultimately limit the AIR with the considered Gaussian receiver. In the weakly nonlinear (around the optimum launch power) and in the highly nonlinear regions, the functional form of the MB constrains the AIR. Slightly better

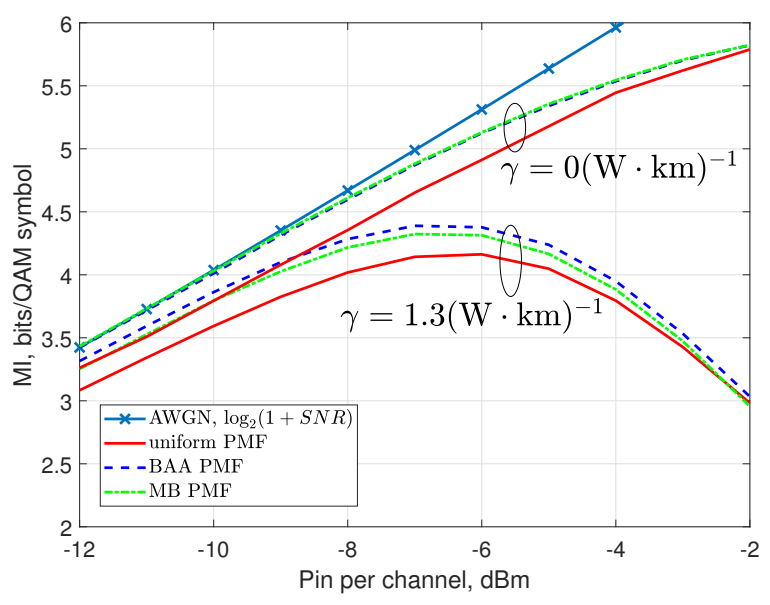

Fig. 3. AIRs on a simple 20x100km fiber link with 5x16 GBd WDM channels, compared to the AIRs on an AWGN channel with the same ASE noise variance.

performance can be achieved by a general 64-D optimization of the PMF through the modified BAA.

\section{F. Measure of Gaussianity}

Outside of the simple examples given in Sections II-D and II-E, PS for optical fiber communications is not well understood. This is especially the case for communication techniques not yet deployed outside of research labs, such as the mid-link OPC scenario focus of this paper. On the other hand, the AWGN channel model is extremely widespread and is applied to much success as an auxiliary channel even in cases where the noise is not strictly Gaussian, as e.g. in optical fiber communications. It is tractable, very well understood, near-optimal signal shapes are known and powerful receivers and DSP techniques have been developed for it. It can thus be attractive to use the AWGN assumptions to non-standard channels, as the ones considered in this paper. In order quantify the level of Gaussianity of the channel and the extent of the validity of this assumption, and thus predict the performance of AWGN-optimized signal shapes (e.g. the MB PMF), the Kullback-Leibler distance (KLD) is employed in this work. The KLD is a measure of distance between two probability distributions and is defined as

$$
D(P \| Q)=\sum_{x \in \mathcal{X}} p(x) \log _{2} \frac{p(x)}{q(x)},
$$

where $P$ and $Q$ are some valid distributions of $X$. In this work, once the sequences $X$ and $Y$ have been generated (either from simulations of the system or experimentally), the additive noise model is employed and the receiver noise is estimated $N=Y-X$. The noise signal is normalized to unit power and a 2-D histogram $P_{N}^{\text {hist }}$ is generated with 100 bins in each $I$ and $Q$ dimensions. A true Gaussian process is then used to generate the signal $\hat{N} \sim \mathcal{N}(0,1)$, which is then quantized to the same bins as the signal $N$. The $\operatorname{KLD} D(N \| \hat{N})$ is then applied to estimate the Gaussianity of the signal $N$ up to a constant, which depends on the parametrization of the histogram binning. It will thus provide a fair estimate of the relative Gaussianity of different noise signals. 
Consequently, channels with noise which is assumed to be white and additive and is "more" Gaussian than others can be expected to benefit from the AWGN-optimal PMF, and viceversa $^{2}$

\section{OPTICAL PHASE CONJUGATION-BASED NONLINEARITY COMPENSATION}

Among the several nonlinearity compensation techniques, OPC presents advantages both in terms of being compatible with WDM transmission, i.e. being able to compensate for both intra and inter-channel nonlinearity, as well as exploiting the near-instantaneous Kerr effect for optical signal processing leading to minimal additional processing latency. The key principle behind the nonlinearity compensation capabilities of OPC is based on the Hermitian structure of the NLSE governing waveform propagation through the fiber and shown in (11).

$$
\frac{\partial A}{\partial z}=-i \frac{1}{2} \beta_{2} \frac{\partial^{2} A}{\partial t^{2}}+\frac{1}{6} \beta_{3} \frac{\partial^{3} A}{\partial t^{3}}-\frac{\alpha}{2} A+i \gamma|A|^{2} A .
$$

In (11) $\alpha, \beta_{2}, \beta_{3}$ and $\gamma$ represent the loss, second order dispersion, third order dispersion and nonlinear coefficients of the fiber, respectively. Considering the most common scenario of an OPC subsystem placed in the middle of a homogeneous transmission link, scenario commonly referred to as mid-link spectral inversion or mid-link OPC, conjugating the WDM signal band is equivalent to inverting the sign of second order dispersion and Kerr nonlinearity within the NLSE (see (12))

$$
\frac{\partial A^{*}}{\partial z}=i \frac{1}{2} \beta_{2} \frac{\partial^{2} A^{*}}{\partial t^{2}}+\frac{1}{6} \beta_{3} \frac{\partial^{3} A^{*}}{\partial t^{3}}-\frac{\alpha}{2} A^{*}-i \gamma|A|^{2} A^{*} .
$$

The sign inversion results in the cancellation of the dispersion and nonlinear distortion accumulated during propagation through the first half of the link simply by further transmitting the conjugated signal on the second half. If ideal compensation with mid-link spectral inversion is achieved and assuming zero parametrically amplified noise and a certain degree of uniformity in the spans, the original receiver $S N R_{0}$ (without nonlinearity compensation) is increased by $1.27 \cdot S N R_{0}^{2 / 3}$, corresponding to a transmission reach enhancement of 1.17 . $S N R_{0}^{1 / 3}$ regardless of the distance[5], [30].

However, in practical transmission links the improvement becomes directly related to the degree of dispersion and power symmetry with respect to the OPC position [31], [32], with the target being identical powers at exactly opposite values of accumulated dispersion. This is generally difficult to achieve because of fiber loss which effectively breaks down the symmetry of dispersion uncompensated EDFA-amplified systems, as shown in Figure 4(a). In this case, the signal exhibits high power at low accumulated dispersion on the left side, and high power at high accumulated dispersion on the right side of the OPC. Consequently, the nonlinearity compensation is poor and the gains are limited. The OPC symmetry can be significantly boosted if distributed Raman amplification is employed [36], [37] as can be seen in Figure 4(b). While

\footnotetext{
${ }^{2}$ The KLD can also be used to quantify the effectiveness of auxiliary channel functions. It can be proven that Eq. (4) turns into equality iff $p(x)=q(x)$ for all $x \in \mathcal{X}$, i.e. $D(P \| Q)=0$ [42].
}

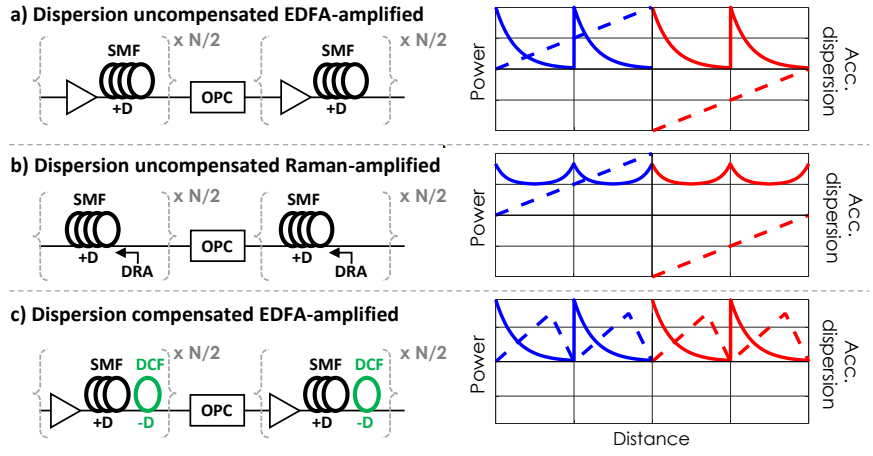

Fig. 4. Power and dispersion symmetry examples for typical optical links with mid-link OPC. Solid lines: power evolution; Dashed lines: dispersion evolution as a function of distance.

that may be of interest for new links, it would require a major infrastructural change for deployed links. Alternatively to addressing the power profile, the symmetry can be increased by dispersion management, i.e. considering legacy dispersion compensated links, as can be seen in Figure 4(c). Although accumulated dispersion remains positive for the entire link, it is close to zero for all high power regions, where most of the nonlinearity is induced. Consequently, the symmetry is approximately preserved in the regions where it matters most. Given its practical relevance and the high expected gains provided by OPC-based nonlinearity compensation, this is the transmission system considered in this work to exemplify the interplay between constellation shaping and OPC.

It is noted that in a practical network, precise placement of the OPC stage may not always be possible. As mentioned in the introduction, partial improvement can also be achieved when deviating quite significantly from the mid-link condition [56]. Furthermore, the use of multi OPC stages within a link can further boost the performance gain in practical scenarios, especially for links where polarization mode dispersion (PMD) becomes significant. In such a case, the SNR improvement has been estimated to scale with the square root of the number of OPC stages employed [5], [57].

\section{SignAl SHAPE- AND CHANNEL-TRANSPARENT DIGITAL SIGNAL PROCESSING}

The DSP design is critical to maximizing the AIR. Any DSP is considered processing of the received analog signal, and thus cannot increase [53] and in practice reduces the MI. It is however necessary to preprocess prior to detection in order for low-complexity, memoryless symbol detection to be accurate. The DSP applied in the experimental part of this paper (replaced by a simplified version for simulation, as discussed in Section V) is designed to be as transparent as possible to the signal shape in order to ensure fairness of comparison of AIRs. The DSP chain is based on pilot symbols interleaved with the constellation-shaped symbols, and is described in details in [22], [58], [59], [60], [61], [62]. Similar DSP chains have also been proposed for highspeed transceivers in a variety of publications [63], [64] and demonstrated to be viable for modulation format transparent applications [47]. For completeness, the DSP chain is depicted 


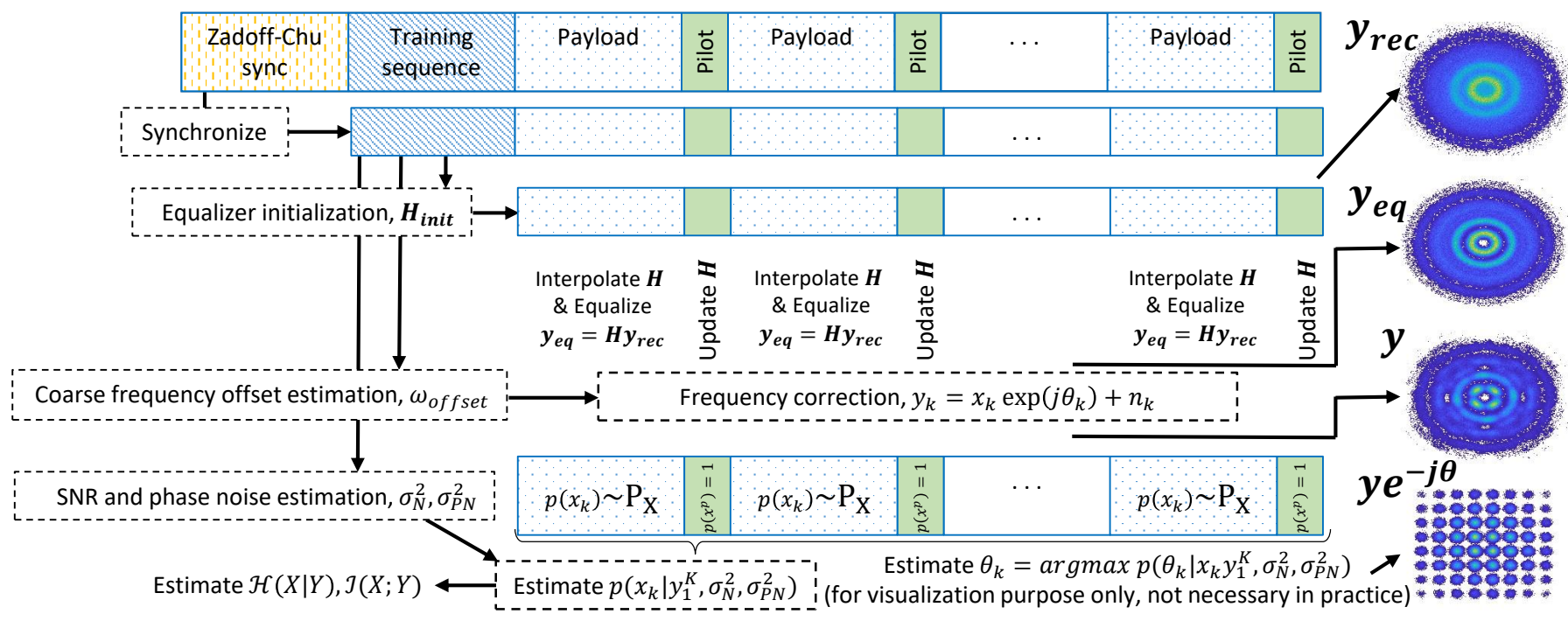

Fig. 5. Modulation-format transparent digital signal processing chain. Example histograms of the processed signal is given on the right after each stage of the chain, denoted as $y_{r e c}, y_{e q}, y$, and $y \cdot e^{-j \theta}$ for the signal before the DSP chain, after equalization, after FO compensation and after phase noise correction, respectively. The phase noise tracking algorithm exploits the knowledge that during payload symbols periods, the transmitted symbols are distributed according to the constellation PMF $p(x) \sim P_{X}$, while during pilot symbol periods, the distribution is an indicator function with $p\left(x^{p}\right)=1$ for the pilot symbol and $p(x)=0$ elsewhere.

in Fig. 5, and summarized below. The pre-facing DSP functions applied in this work such as low-pass filtering, Gardner re-sampling and $\mathrm{CD}$ compensation in frequency domain are considered standard and are not elaborated on.

\section{A. Training stage}

A Zadoff-Chu (ZC) sequence [65] of length 101 is inserted at the beginning of transmission serving as a frame synchronization sequence. The ZC sequence is a constant amplitude zero-autocorrelation waveform which is very effective for synchronization in the presence of various channel and transceiver impairments, particularly for heterodyne detection with free-running local oscillator. The $\mathrm{ZC}$ sequence is followed by a training sequence, which has the same distribution as the payload, and is available at the receiver. In this work, the training sequence is randomly generated. In practice, properly designed training sequence can potentially reduce its minimum required length to achieve the following:

1) Initialize the equalizer by applying the data-aided multimodulus algorithm (MMA) [66]

2) Obtain a coarse frequency offset (FO) using a peak search in the frequency domain of the signal $y_{b e a t_{k}}=$ $y_{\text {train }_{k}} \cdot x_{\text {train }_{k}}^{*}$, where $y_{\text {train }_{k}}$ is the received signal in the training period and $x_{\text {train }}^{*}$ is the complex conjugated training sequence [67].

3) Extract the Gaussian noise and phase noise process variances.

After equalization and frequency offset correction, the signal is assumed to be modeled as a Wiener phase noise channel

$$
\begin{array}{r}
y_{k}=x_{k} \cdot \exp j \theta_{k}+n_{k}, \\
\theta_{k}=\theta_{k-1}+v_{k}, \\
V \sim \mathcal{N}\left(0, \sigma_{P N}^{2}\right),
\end{array}
$$

where $\theta_{k}$ is the phase noise sample at time $k$ and originates in a Gaussian random walk [61]. Using this model, the parameters $\sigma_{N}^{2}$ and $\sigma_{P N}^{2}$ can be extracted as

$$
\begin{array}{r}
\hat{\theta}_{k}=\angle \sum_{l=k-L / 2}^{k+L / 2} y_{\text {train }_{e q_{l}}} \cdot x_{\text {train }_{l}}^{*}, \\
\hat{y}_{\text {train }_{\text {eq }}}=y_{\text {train }_{e q_{k}}} \cdot e^{-j \hat{\theta}_{k}}, \\
\sigma_{P N}^{2}=\mathbb{E}\left[\left(\hat{\Theta}_{k}-\hat{\Theta}_{k-1}\right)^{2}\right], \\
\sigma_{N}^{2}=\mathbb{E}_{k}\left[\left|\hat{y}_{\text {train }_{\text {eq }}}-x_{\text {train }_{k}}\right|^{2}\right],
\end{array}
$$

where $\angle$ is the angle function and $y_{\text {train }_{e q}}$ is the equalized sequence received during the training period. The choice on the window length $L$ used for extraction of the phase noise sample has an influence on the accuracy and on the subsequent noise variance estimation, and is in the ideal case optimized for final performance. At the operating SNR region in this work, $L=50$ was found to be a good balance between accuracy of the phase noise estimation (16) and accuracy of the noise variance estimation (19).

\section{B. Performance measurement stage}

In order to perform adaptive equalization, which is necessary in coherent systems due to polarization drifts and PMD, pilot symbols are inserted periodically in the sequence of transmitted symbols. In this work, the pilots are chosen to be 4QAM in order to apply the simple constant modulus algorithm (CMA) for equalization. The equalizer is updated at each pilot position according to the CMA update rule [68], which tries to force the received symbols on the unit circle. Different variants then exist for equalization of the payload symbols. For example, sample-and-hold strategy can be chosen as a low-complexity solution, or a decision-directed MMA can 
be applied at the tracking stage. The latter suffers performance penalty for probabilistically shaped signals [69]. Thus, in this work, we chose to interpolate the equalizer taps between pilot positions in order to avoid MMA and make the equalizer modulation format transparent [59].

Any remaining frequency offset after the coarse FO estimation is assumed minor and incorporated into the phase noise variance $\sigma_{P N}^{2}$. In cases where the FO changes too fast between training sequences, the pilots can be used to track and correct the FO in a similar frequency domain peak search manner at a slight complexity expense. The latter option also alleviates the workload on the phase noise compensation stage.

The phase noise compensation algorithm from [60] which is applied in this work does not perform the classical estimation and correction steps. Instead, the algorithm estimates the posterior probabilities of the transmitted symbols $p\left(x_{k} \mid y_{1}^{k}, \sigma_{N}^{2}, \sigma_{P N}^{2}\right)$ given the observations and estimated parameters directly in the presence of the phase noise. While estimating the posteriors, the constellation PMF is taken into account in order to improve the detection in cases of PS. The posteriors are estimated using a forward-backward recursionbased belief propagation algorithm in a sliding window fashion going over the received sequence. Due to lengthy derivation and cumbersome notation, details of the algorithm are omitted here. The complete recursions can be found in [60] and their sliding window length optimization in [62].

The phase noise samples $\theta_{k}$ are not explicitly estimated, but rather their distribution at each time $p_{\Theta_{k}}$. If required, for example for visualization purposes as in Fig. 5, the phase noise samples can be estimated from the phase noise distribution as $\theta_{k}=\arg \max p\left(\theta_{k}\right)$, and then used to plot a phase noisefree signal $y e^{-j \theta}$. However, this step is not a requirement for either the estimation of the entropy $\mathcal{H}(X \mid Y)$, or in practice for detection. Observe that the detector is interested in the posterior probabilities rather than in the actual phase noise signal.

\section{Universal PMF optimization algorithm}

Optimization of the PMF is an $|\mathcal{X}|-\mathrm{D}$, generally non-convex problem, except for special cases, such as the above-mentioned AWGN channel, for which a unique optimum can be found. In this work, a greedy optimization algorithm [41] is adopted that improves on the stability, accuracy and convergence speed of the BAA [26] by assuming identical probability of occurrence of constellation points with equal amplitude, effectively reducing the dimensionality of the optimization problem. The basic idea of greedy optimizers is to assume that each dimension can be optimized locally and then iterate between dimensions. A summary of the adopted greedy approach, which was also applied in [41], is given in Algorithm 1.

The universality stems from the fact that on Step 8, any channel, simulation or experimentally based can be applied. The examples of interest to this work are 1) the channel model based on solving the NLSE via the split-step Fourier method (SSFM); and 2) an experimental channel. For the former option, $y$ is the sequence after low-pass filter, resampling and $\mathrm{CD}$ compensation are performed, while in the latter case,

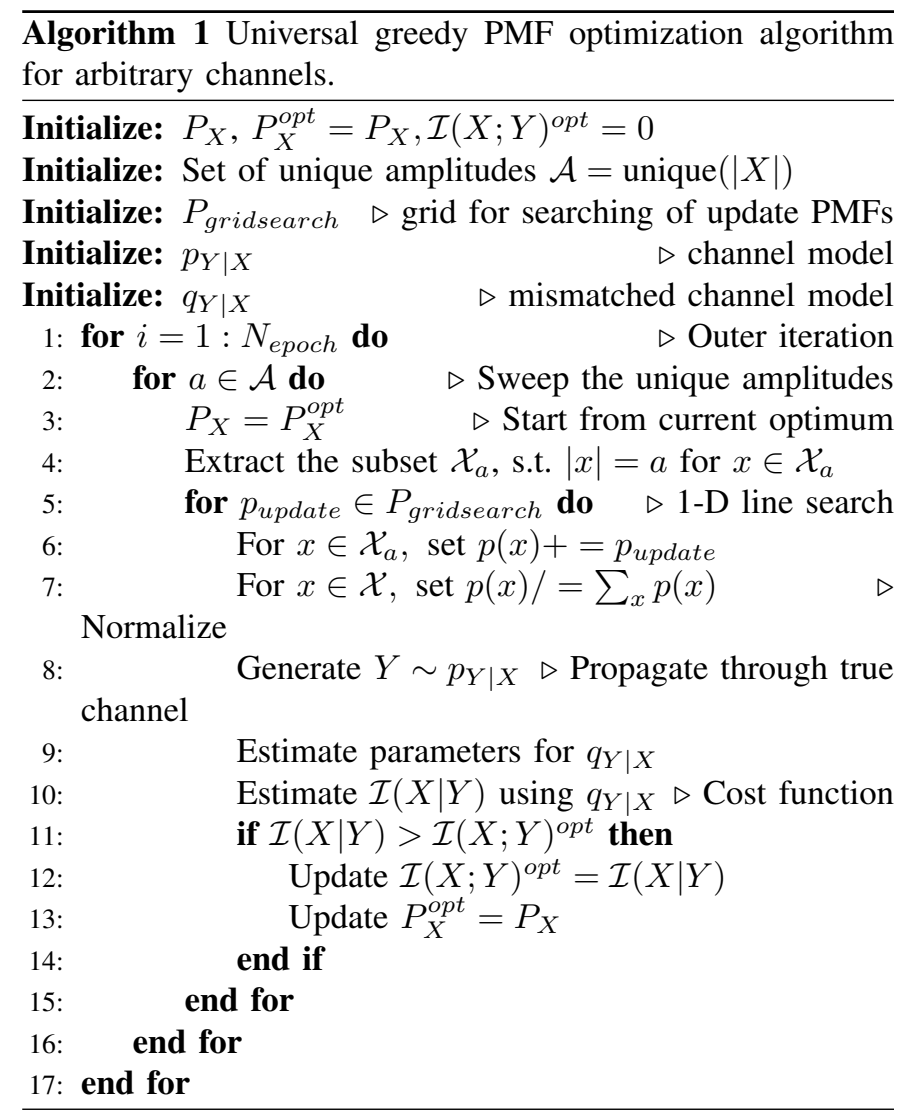

the DSP chain is also part of the effective channel as seen by the symbol detector. Clearly, both cases include a certain amount of processing, which would be difficult to incorporate in a rigorous theoretical optimization algorithm, e.g. one that requires differentiation. By including all relevant processing blocks in the effective channel model $p_{Y \mid X}$ used for the optimization in Algorithm 1, we guarantee that the optimized PMF is explicitly targeting the practical system instead of an idealized one, which might be easier to optimize.

The sub-optimality of the algorithm is mainly related to the fact that in e.g. the optical fiber channel, the true distribution $p_{Y \mid X}$ and the corresponding channel SNR depend on the input $P_{X}$ through the Kerr nonlinearity. An optimum solution is thus a compromise between a PMF, which is beneficial in the presence of Gaussian noise and a PMF resilient to nonlinearities. Ultimately, the shape of the optimum solution is not obvious, with some indications that a good compromise is achieved by a ring-like constellation [4], [70], [71], [28]. The presence of nonlinearity compensation inherently changes the balance of nonlinear and linear noise in the system. Optimal solutions in this case and gains thereof are also not obvious, with several experiments demonstrating that the shaping gains might add up to the nonlinearity compensation gains: constellation shaping combined with DBP was demonstrated in [72], whereas constellation shaping combined with OPC was demonstrated in [26], [41].

\section{Simulation Results}

The system under test is given in Fig. 6. For each WDM channel, random symbols are generated independently from 


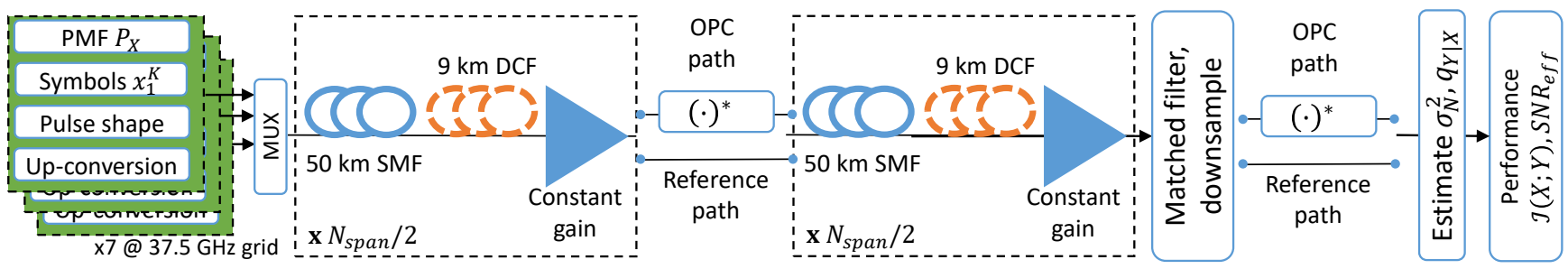

Fig. 6. Simulation block diagram.

TABLE I

FIBER PARAMETERS USED IN THE SIMULATION AND THE EXPERIMENT. HNLF USED ONLY IN EXPERIMENT.

\begin{tabular}{c||c|c|c} 
& SSMF & DCF & HNLF \\
\hline Loss $\alpha, \mathrm{dB} / \mathrm{km}$ & 0.2 & 0.5 & 0.82 \\
\hline Nonlinear coefficient $\gamma,(\mathrm{W} \cdot \mathrm{km})^{-1}$ & 1.3 & 5.7 & 9.7 \\
\hline Dispersion $D @ 1550 \mathrm{~nm},\left(\mathrm{ps} \cdot(\mathrm{nm} \cdot \mathrm{km})^{-1}\right.$ & 17 & -95 & 0.52 \\
\hline \hline
\end{tabular}

a given PMF. The symbol sequence is pulse shaped using a square-root raised cosine filter with a roll-off of 0.01. Seven WDM channels are simulated with $32 \mathrm{GBd}$ in each and 37.5 $\mathrm{GHz}$ channel spacing. The WDM signal is propagated using a SSFM channel model through a multi-span EDFA-amplified link. The SSFM is implemented as asymmetric and applying an adaptive step size by limiting the maximum allowed phase rotation [73]. Each span consists of $50 \mathrm{~km}$ of SSMF followed by $\approx 9 \mathrm{~km}$ of dispersion compensating fiber (DCF) providing ideal dispersion compensation, with parameters given in $\mathrm{Ta}-$ ble I. Zero dispersion slope is assumed for the SSMF and DCF. Nonlinearity compensation is achieved by assuming an ideal OPC stage, consisting of signal conjugation at the middle of the link. At the receiver side, matched filtering is performed, followed by down-sampling and performance estimation. In the simulation case, ideal lasers with neither amplitude nor phase noise are assumed, and identical oversampling factor of 56 (ensuring the Nyquist criterion is satisfied for the case of 7 channels, and taking into account the potential bandwidth expansion and the non-zero channel spacing) is applied at both the transmitter and receiver. The DSP chain from Fig. 5 is thus bypassed, with the exception of the estimation of the variance of the Gaussian auxiliary function $\sigma_{N}^{2}$ (19). Algorithm 1 is then applied to optimize the PMF. The optimized and uniform PMFs are compared to: 1) the MB PMF which is optimal for an AWGN channel with an SNR, equivalent to the effective SNR of uniform input PMF; and 2) a MB PMF which is optimized with a 1-D line search for the optimal parameter $\lambda$ as mentioned above. The AIR is estimated from a sequence of $K=500.000$ independent symbols in each case, also at each iteration of Algorithm 1. With the exception of Fig. 11, the central channel of the WDM signal is studied as a worst case scenario. In all cases, including Section VI, performance is measured as a function of the launch power per channel.

In Fig. 7, the received $S N R_{\text {eff }}$ is given for each PMF as a function of the launch power for the reference and OPC paths, and in Fig. 8, the AIRs of Gaussian auxiliary channel receiver are given. When standard uniform PMF is considered, midlink OPC delivers $\approx 1.1 \mathrm{~dB}$ of gain in effective SNR, which
TABLE II

OPTIMUM VALUES OF $\lambda$ FOR THE STUDIED MB PMFS AT THE RESPECTIVE OPTIMUM LAUNCH POWER. HIGHER $\lambda$ INDICATES MORE PEAKY PMF, AS SEEN FROM (8).

\begin{tabular}{c||c|c} 
& based on $S N R_{\text {eff }}$ & line search \\
\hline with OPC & 1.6 & 1.4 \\
\hline w/o OPC & 1.9 & 0.4 \\
\hline \hline
\end{tabular}

translates into $\approx 0.35$ bits/QAM symbol of AIR. The MB PMF optimized for an AWGN channel results in a degraded $S N R_{e f f}$, and ultimately a penalty in AIR. This penalty can be mostly overcome by optimizing the MB PMF through a line search for the parameter $\lambda$. The shaping gains can be increased further to $\approx 0.07$ bits/QAM symbol by applying Algorithm 1 .

When OPC is applied, the received $S N R_{\text {eff }}$ becomes less susceptible to highly-peaky constellation PMFs, allowing for well-establshed shaping strategies e.g. from the AWGN channel (as in this paper) to achieve most of the available shaping gain up to the weakly nonlinear region. The shaping gain can correspondingly be increased w.r.t. the case without $\mathrm{OPC}$ to $\approx 0.14$ and $\approx 0.16$ bits/QAM symbol with $\mathrm{MB} \mathrm{PMF}$ and by applying Algorithm 1, respectively. When the launch power is increased to the nonlinear region, the penalty of MB PMFs appears. The optimum values of $\lambda$ for the MB optimization at the respective optimum launch power are given in Table II for the cases with and without OPC.

The optimal PMFs for the cases without and with OPC at the respective optimal launch powers are given in Fig. 9. In the former case, the PMF exhibits suppressed inner points and resembles the ripple constellation, proposed in [70] for continuous input distributions, as well as the constellations, optimized for an unrepeatered transmission in [28]. In the latter case, the PMF is qualitatively similar to the MB PMF.

The noise statistics of dispersion compensated fiber transmission are further studied using the KLD between the distribution of the estimated noise variable $N=Y-X$ and a Gaussian distribution with the same variance as described in Section II-F for the cases with and without OPC in Fig. 10. In the low-power, linear region of transmission, the noise statistics are independent of the PMF and have a constant KLD to the continuous Gaussian distribution. The non-zero KLD is attributed to the discretization of the theoretically continuous noise. At the optimal launch power without OPC of $2 \mathrm{dBm}$, in contrast to the uniform case, the distribution of the noise for MB PMF input begins to significantly diverge from the Gaussian distribution. In addition to the overall reduced SNR, this fact degrades the performance of the Gaussian auxiliary 


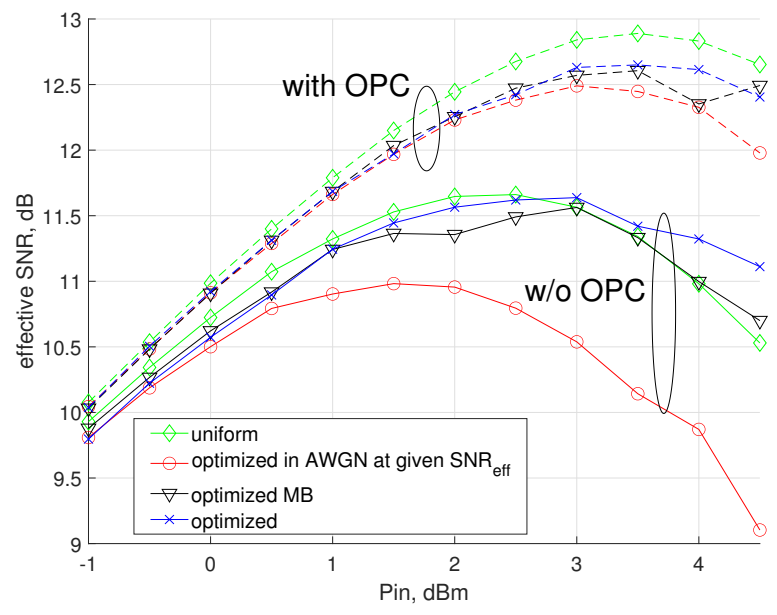

Fig. 7. Received $S N R_{e f f}$ for the studied optimization schemes. Dashed lines: with OPC; solid lines: w/o OPC. The AWGN-optimal MB PMF results in $\approx 0.5 \mathrm{~dB}$ SNR degradation due to the high peak power of the constellation. This effect is diminished when applying OPC due to the nonlinearity compensation. OPC results in $\approx 1.1 \mathrm{~dB}$ of improvement for uniform PMF

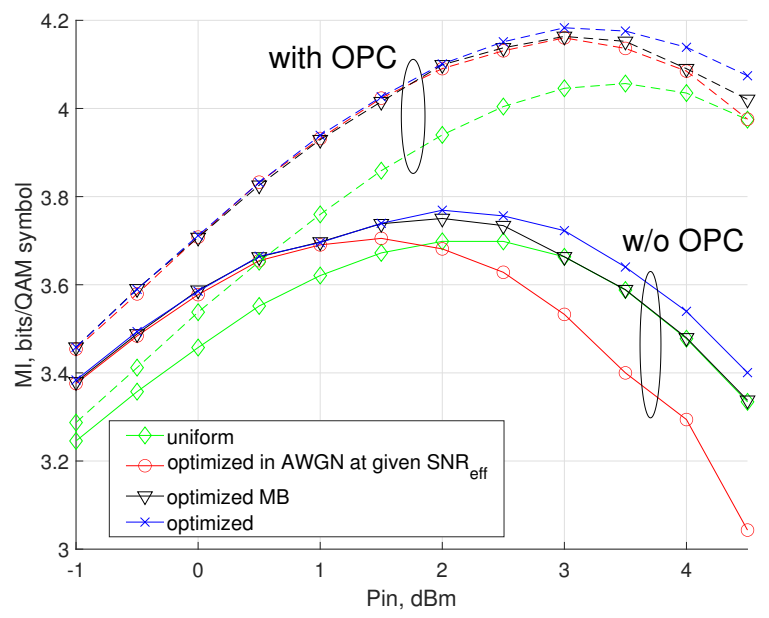

Fig. 8. AIR of the studied optimization schemes. Dashed lines: with OPC; solid lines: w/o OPC. Without nonlinearity compensation, proper optimization is required in order to see shaping gains. When OPC is applied, shaping gains are more straight-forward to obtain. OPC results in $\approx 0.35 \mathrm{bits} / \mathrm{QAM}$ symbol of improvement for uniform PMF. Shaping with Algorithm 1 results in $\approx$ 0.07 bits/QAM symbol of improvement w.r.t. uniform PMF without OPC and $\approx 0.16$ bits/QAM symbol with OPC.

channel, resulting in the poor performance of the AWGNoptimal MB PMF. When OPC is applied, the noise remains Gaussian further into the nonlinear region including at the respective optimal launch power of $3 \mathrm{dBm}$. This explains the near-optimality of Gaussian channel-like transmission strategies, including PMF and auxiliary channel choices. The convergence of the noise back to Gaussian distribution in all cases at extreme launch power may be attributed to dispersed extreme nonlinearities which are perceived as noise in the memoryless receiver, or even spectral broadening. This is left for future work to confirm.

Finally in this section, the robustness of the designed PMFs is studied by applying the PMFs, optimized for the central channel to the other channels in the WDM signal. Their per-

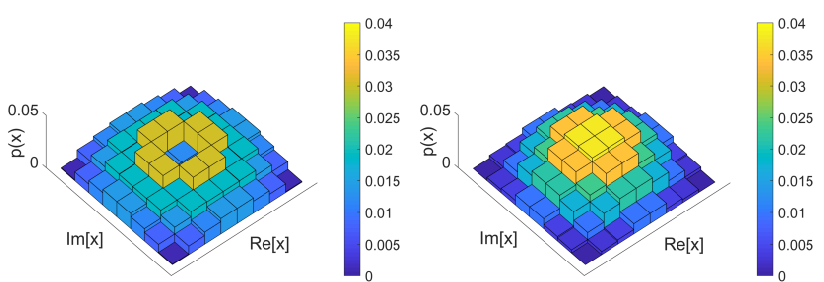

Fig. 9. Optimal PMFs without OPC (left) and with OPC (right) at the respective optimal launch powers. In the case without OPC, a ripple-like [70] PMF is preferred, while in the OPC case, a MB shape is obtained.

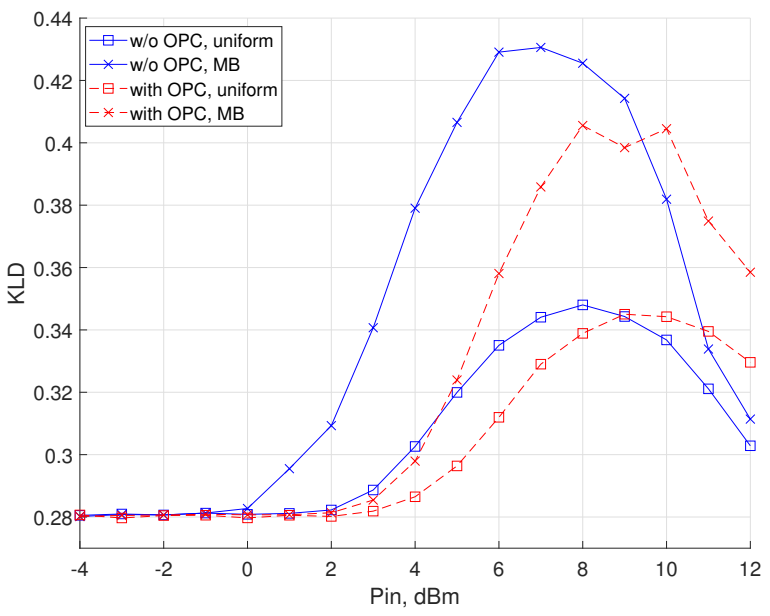

Fig. 10. Estimated noise Gaussianity through the KLD to a true Gaussian distribution. When OPC is applied, the noise is relatively Gaussian until the optimal launch power $(\approx 3 \mathrm{dBm})$. For the DCF-based link without OPC, the noise is highly non-Gaussian already in the weakly nonlinear region for the MB input.

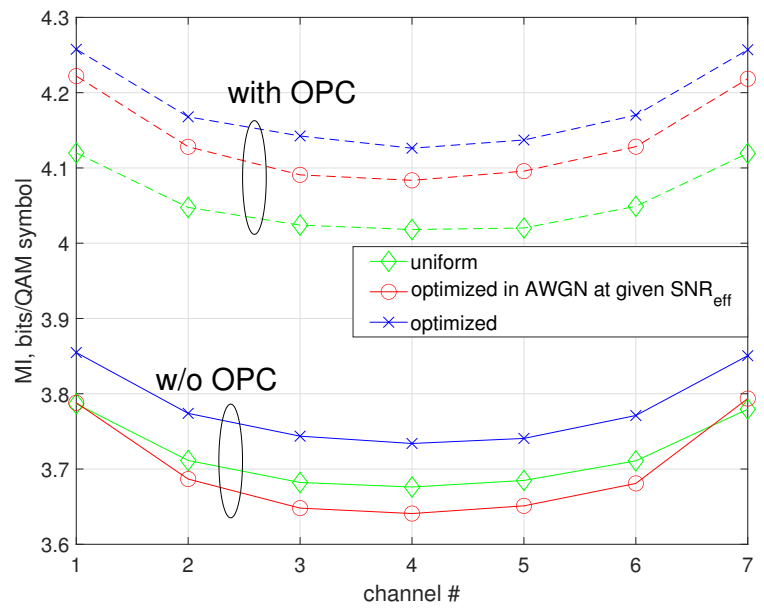

Fig. 11. AIR per WDM channel with the PMFs, optimized for the central channel. Dashed lines: with OPC; solid lines: w/o OPC. Channels are ordered from low to high frequency. The optimized PMF is robust to different WDM channels. At the WDM signal edge, nonlinearities are less pronounced, improving the AIR and making the MB slightly more effective.

formance is shown in Fig. 11 at the respective optimal launch power with and without OPC. Channels at the signal edge perform slightly better due to fewer interfering channels on one side of the spectrum and thus lower nonlinear interference. 
The latter also has impact on the effectiveness of the MB PMF, which in the case without OPC is now slightly better w.r.t. the uniform distribution. The PMF which was designed for the central channel achieves virtually the same shaping gain across the band. It is noted that the band edge effect is only marginally relevant for fully-loaded systems.

\section{EXPERIMENTAL DEMONSTRATION}

The experimental setup is illustrated in Figure 12. The transmission signal used is a $32 \mathrm{GBd}$ with root-raised cosine pulse shapes at 0.01 roll-off. The $32 \mathrm{GBd}$ sequence was modulated onto carrier lasers (external cavity lasers (ECLs) with $\approx 10 \mathrm{kHz}$ linewidth) using a 4-channel arbitrary waveform generator (AWG) $(64 \mathrm{GSa} / \mathrm{s})$ driving two I/Q modulators with independent data on each - one for odd-numbered and one for even-numbered channels. The central channel is centered at $1540 \mathrm{~nm}$. The WDM signal is then replicated on the orthogonal polarization, with a delay to ensure decorrelation between the polarizations.

The optical fiber channel consisted of two loops of dispersion compensated links with OPC in between. The first loop is composed of four spans: $50.5 \mathrm{~km}$ SSMF, $8.6 \mathrm{~km} \mathrm{DCF,} 44 \mathrm{~km}$ SSMF and $6 \mathrm{~km}$ DCF. Likewise is the second loop composed of four spans with: $50.5 \mathrm{~km}$ SSMF, $7.2 \mathrm{~km}$ DCF, $44 \mathrm{~km}$ SSMF and $6.9 \mathrm{~km}$ DCF. Any residual dispersion is compensated by the adaptive equalizer. Ten circulations were allowed in each loop, resulting in a total distance of $\approx 2176 \mathrm{~km}$. The OPC stage is based on a standard single-pump FWM setup in a $254 \mathrm{~m}$ long highly-nonlinear fiber (HNLF) with stable phasematching for improved nonlinear efficiency (HNLF-SPINE) characterized by the parameters of Table I and with a zerodispersion wavelength at $1544 \mathrm{~nm}$. Dual-polarization conjugation is achieved by using a polarization-diversity scheme where two orthogonal signal polarizations counter propagate within the HNLF. The FWM pump is placed at $1545 \mathrm{~nm}$ and it is generated by amplifying a $10-\mathrm{kHz}$ ECL with high OSNR in order to minimize the phase and amplitude noise transfer from the pump to the conjugate signal (idler). Following energy conservation, the FWM idler is then generated around $1550 \mathrm{~nm}$ and after recombining the two polarizations, pump and signal bands are suppressed using optical filters and the 1550-nm conjugate band is further propagated through the second half of the transmission link.

On the receiving end, the central channel is acquired with an $80 \mathrm{GSa} / \mathrm{s}$ digital oscilloscope, the signal is digitally processed using the DSP chain from Section IV and the MI is estimated using a Gaussian auxiliary channel. Algorithm 1 is then applied experimentally. Effectively, an experimental measurement is taken for each candidate PMF in the bruteforce optimization of the probability of occurrence of the amplitudes of the constellation. This measurement then drives PMF updates in Algorithm 1. It is thus critical that the experimental test-bed is as stable as possible in order to remove the effects of drifts, which can potentially mask the shaping gain and/or lead to globally sub-optimal solutions. In order to improve the convergence speed and mitigate this problem, the PMF is initialized to a simulation-based optimum, where the simulation followed the parameters of the experimental setup. After initialization, optimization is resumed experimentally in order to tailor the PMF to the experimental setup.

\section{A. Results}

In Fig. 13, the received $S N R_{e f f}$ is given for each PMF as a function of the launch power for the reference and OPC paths, and in Fig. 8, the AIRs of the Gaussian auxiliary channel receiver are given. In each case, 100.000 independent symbols are used to estimate the AIR and the SNR. When standard uniform PMF is considered, mid-link OPC delivers $\approx 1.2 \mathrm{~dB}$ of gain in effective SNR, which translates into $\approx 0.4$ bits/QAM symbol of AIR. This is consistent with the gains reported in other studies of the mid-link OPC-based EDFA-amplifier dispersion compensated transmission systems [5], [26], [74]. Similar to the simulation, the experimental results demonstrate that when OPC is applied, the AWGNoptimized input PMF achieves near-optimal shaping gain of $\approx 0.2$ bits/QAM symbol. Without OPC, the MB PMF is severely penalized in the highly nonlinear regime, but achieves $\approx 0.07$ bits/QAM symbol of gain at the optimal launch power, which was not possible in simulations. This can be attributed to the implementation penalties, which manifest themselves as an extra Gaussian noise source and enhance the Gaussianity of the total noise experienced by the receiver. It should be noted that part of the non-Gaussianity of the noise statistics can be attributed to nonlinear phase noise (NLPN), which is partially tracked and compensated for by the PN tracking algorithm [60], [61]. This fact together with the Gaussian distributed implementation penalty allows the MB PMF to achieve slightly higher shaping gain in the weakly nonlinear region w.r.t. the idealized simulation. Algorithm 1 achieves an improvement of $\approx 0.2$ bits/QAM symbol in both transmission cases.

Building a histogram for the received noise is challenging experimentally due to the requirement of high number of samples for accurate histogram representation. For the results in Fig. 10, 30.000.000 symbols were used. Gathering that many consistent samples experimentally is challenging due to experimental drifts. Instead, the effect of the MB PMF on the received noise statistics is measured indirectly through the severity of the phase noise, experienced by the receiver. In particular, the standard deviation $\sigma_{P N}$ estimated as in Section IV-A. The phase noise experienced by the receiver is a combination of laser phase noise and NLPN [60], [61]. In Fig. 15, the estimated values of $\sigma_{P N}$ are given for all studied input PMFs. The MB PMF results in an increased phase noise, which is part of the reason for its poor performance in the nonlinear region without OPC. When OPC is applied, the phase noise statistics are far less dependent on the PMF at least in the weakly nonlinear region, making the MB PMF selected for an AWGN channel with that effective SNR near-optimal. It can also be noted that the laser phase noise contribution can be estimated as [60] $\sigma_{P N}^{\text {lasers }}=\sqrt{2 \cdot \pi \cdot \Delta_{f} T_{s}}$, where $T_{s}$ is the symbol period and $\Delta_{f}$ is the combined linewidth of all lasers interacting with the signal. Considering $\approx 10 \mathrm{kHz}$ linewidth of the ECLs, $\sigma_{P N}^{\text {lasers }}$ can be estimated to be $\approx 2 \cdot 10^{-3}$ in the 


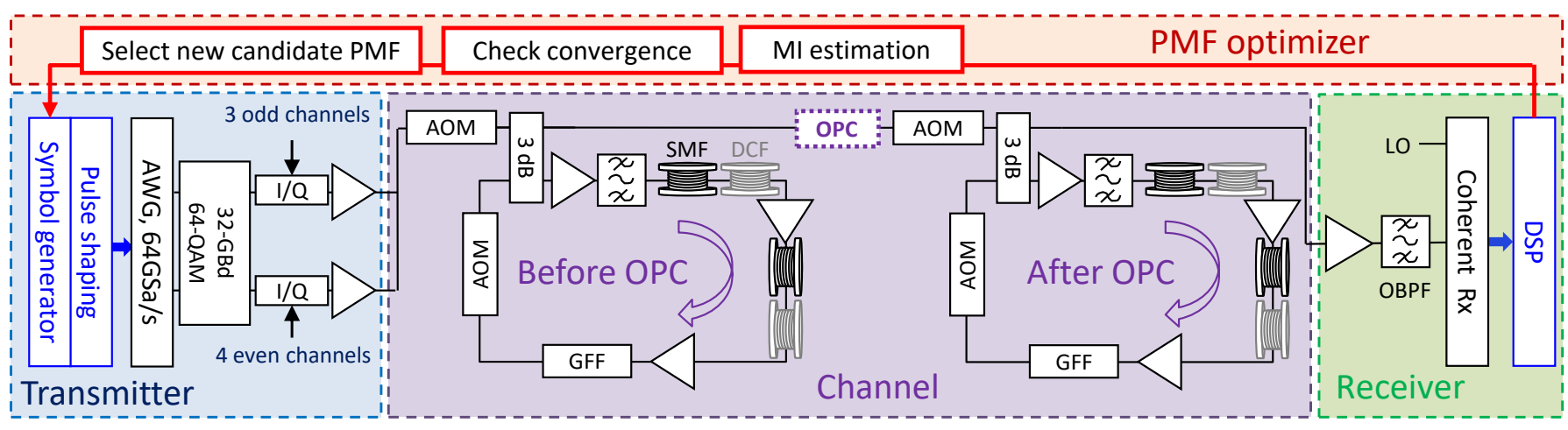

AWG-arbitrary waveform generator, AOM-acousto-optic modulator, GFF-gain flattening filter, OBPF-optical band-pass filter, LO-local oscillator

Fig. 12. Experimental setup for the mid-link OPC-based nonlinearity compensation.

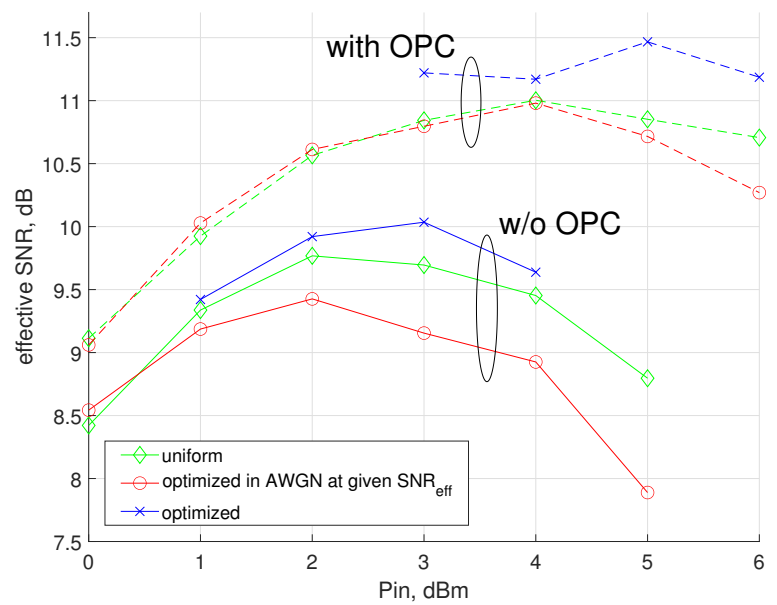

Fig. 13. Received experimental $S N R_{\text {eff }}$ for the studied optimization schemes. Dashed lines: with OPC; solid lines: w/o OPC. Similar to simulations, the AWGN-optimal MB PMF results in SNR degradation due to the high peak power of the constellation. This effect is diminished when applying OPC due to the nonlinearity compensation. OPC results in $\approx 1.2$ $\mathrm{dB}$ of improvement for uniform PMF.

case without $\mathrm{OPC}$, increased to $\approx 2.8 \cdot 10^{-3}$ in the case with OPC due to the extra laser phase noise transferred from the pump. It is then clear that the NLPN is dominating the case of transmission without $\mathrm{OPC}$, and is largely mitigated by the use of OPC.

Experimentally, only the central channel was acquired and optimized, and its PMF is applied to both the odd and the even channels. Such a sub-optimal decorrelation potentially has the effect of overestimating the nonlinear interference and thus underestimate the performance [75], [76]. Due to the qualitative similarity between the central channel performance in the experiment and simulation, we conclude that this effect is not as pronounced for the chosen system parameters. Similarly, we do not expect the conclusions to deviate for the neighboring WDM channels.

\section{DISCUSSION AND FUTURE WORK}

In this work, a circularly symmetric complex-valued Gaussian auxiliary channel is considered. As mentioned, the PN tracking algorithm allows for part of the non-circularity to

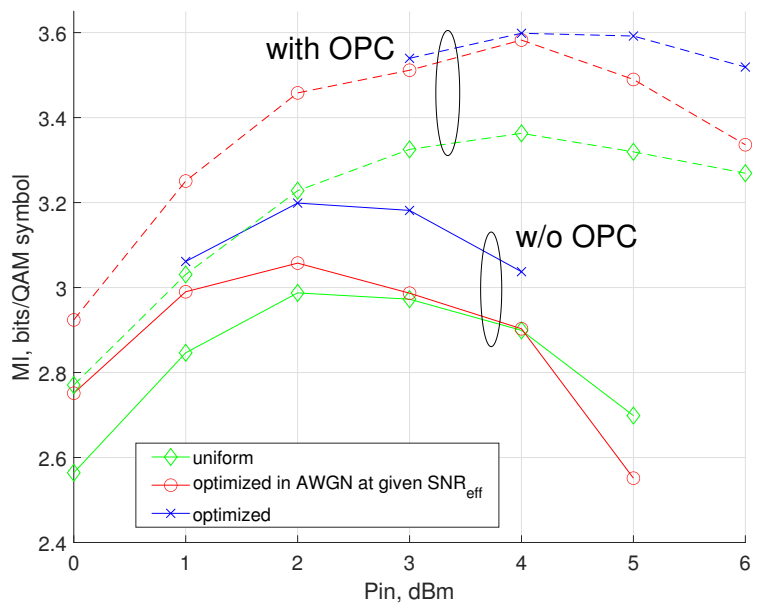

Fig. 14. Experimental AIR of the studied optimization schemes. Dashed lines: with OPC; solid lines: w/o OPC. Similar to simulations, without nonlinearity compensation, proper optimization is required in order to see shaping gains. When OPC is applied, shaping gains are more straight-forward to obtain. OPC results in $\approx 0.4$ bits/QAM symbol of improvement for uniform PMF. Shaping with Algorithm 1 results in $\approx 0.2$ bits/QAM symbol of improvement w.r.t. uniform PMF both with and without OPC.

be compensated for. A correlated Gaussian can potentially further improve the quality of the auxiliary channel [22], [48]. Such a receiver essentially assumes a unique covariance matrix and mean for the noise around each point from the constellation. The likelihood becomes $p_{Y \mid X^{i}}=\mathcal{N}\left(\mu^{\mathbf{i}} ; \boldsymbol{\Sigma}^{\mathbf{i}}\right)$, where $\mathbf{y}=[\operatorname{Re}[y] ; \operatorname{Im}[y]]$ is a $2-D$ real-valued vector and $\mu^{\mathbf{i}}$ and $\boldsymbol{\Sigma}^{\mathbf{i}}$ are the mean and covariance matrix for the likelihood of the $i$-th symbol from the constellation. Training the covariance matrix for each constellation symbol requires a high amount of training points for each symbol, respectively. In cases where probabilistic shaping is applied, the probability of occurrence of some points easily reaches $10^{-5}$ in the optimal PMF. Assuming 100 symbols are required for relatively accurate covariance matrix estimation, a total of 10.000.000 training symbols must be sent on the channel, which is infeasible in a time-limited and stability-sensitive experiment. A correlated Gaussian reception is thus omitted in this work.

For similar reasons, PMF optimization is restricted to a single channel use, i.e. one period in the time domain, and 


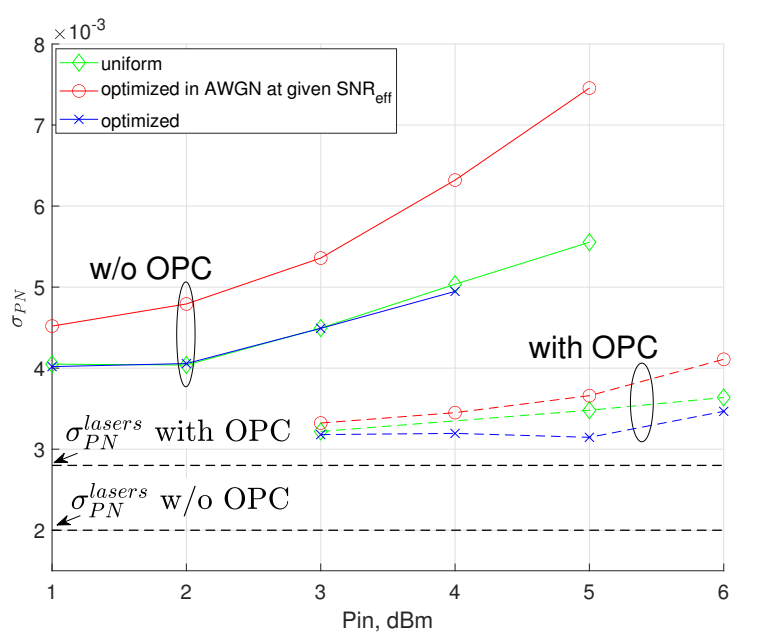

Fig. 15. Estimated phase noise $\sigma_{P N}^{2}$ for the studied input PMFs. Dashed lines: with OPC; solid lines: w/o OPC. When OPC is applied, the PN is less dependent on the input and allows for the AWGN-optimal MB to achieve most of the shaping gains. Furthermore, the NLPN contribution is significantly lower with OPC. Laser linewidth contribution given for reference in both cases with and without OPC.

multi-dimensional shaping through the time, polarization and frequency dimensions are omitted. In this case, the shaping gains in nonlinear optical fibers, including those with OPCbased nonlinearity compensation are rather restricted, and rarely exceed the shaping gains in AWGN channels with similar SNR. Multi-dimensional probabilistic [27], [77] and geometric shaping [29], [78], [79] can be beneficial in combating nonlinearities and provide a shaping gain, which is higher than the ultimate shaping gain for an AWGN channel, but at the cost of increased transmitter and receiver complexity.

Conversely, what was demonstrated in this paper is that at least in dispersion compensated scenarios, OPC-based nonlinearity compensation makes the noise perceived by the receiver "more Gaussian". The other properties of the AWGN, such as being additive and white (implying independence between symbols across all dimensions, namely time, frequency and space) are properties of the chosen auxiliary channel. Estimating the effectiveness of those for a channel with OPC is an important step towards understanding the potential shaping gain for such channels when the auxiliary function is not constrained as in this paper. As demonstrated, the nonlinear phase noise-induced memory is suppressed when OPC is applied, indicating that the independence in time becomes more pronounced. An important consequence of the independence property is that shaping gains would be limited to the ultimate shaping gain of $1.53 \mathrm{~dB}$ for the AWGN channel. This is left for future work to confirm.

\section{CONCLUSION}

Mid-link optical phase conjugation (OPC) was studied as means to compensate nonlinearities in dispersion-compensated fiber links. Some digital aspects of the optimization of the OPC-based link were discussed. In particular, a shaping- and channel-agnostic digital signal processing chain was presented and demonstrated, which allows for arbitrary constellation shapes to be transmitted and for shaping gains to be realized on complicated channels such as the mid-link OPC channel. The proposed DSP chain also allows for fair comparison of modulation formats and their respective probability mass functions (PMFs). The PMF itself was also specially optimized for the dispersion-compensated case and its OPC counterpart by a simple greedy optimization algorithm, which was shown to outperform standard PMF optimization of MaxwellBoltzmann (MB) shapes. The optimization algorithm was demonstrated both through simulations and experimentally for $\mathrm{a} \approx 2000 \mathrm{~km}$ link. The noise characteristics of the system were studied and shown to be highly non-Gaussian for the standard non OPC case, particularly when the peaky MB PMF is applied. On the contrary, mid-link OPC results in 1) a higher received SNR; and 2) Gaussian noise statistics that are relatively independent of the PMF. Both elements contribute to improved performance when standard Gaussian auxiliary channel-based detector is applied. Furthermore, the Gaussianity of the noise in the OPC case allows for the simple MB PMF shape to be applied with near-optimal results, whereas in the standard case, the MB PMF was demonstrated to be sub-optimal.

\section{APPENDIX A}

\section{GLOSSARY OF THE MOST COMMON ABBREVIATIONS}

$\begin{array}{ll}\text { AIR } & \text { Achievable information rate } \\ \text { ASE } & \text { Amplified spontaneous emission } \\ \text { AWG } & \text { Arbitrary waveform generator } \\ \text { AWGN } & \text { Additive white Gaussian noise } \\ \text { BAA } & \text { Blahut-Arimoto algorithm } \\ \text { CD } & \text { Chromatic dispersion } \\ \text { CMA } & \text { Constant modulus algorithm } \\ \text { CS } & \text { Constellation shaping } \\ \text { DCF } & \text { Dispersion compensating fiber } \\ \text { DM } & \text { Distribution matcher } \\ \text { DSP } & \text { Digital signal processing } \\ \text { ECL } & \text { External cavity laser } \\ \text { EDFA } & \text { Erbium doped fiber amplifier } \\ \text { FO } & \text { Frequency offset } \\ \text { FWM } & \text { Four-wave mixing } \\ \text { GS } & \text { Geometric shaping } \\ \text { HNLF } & \text { Highly nonlinear fiber } \\ \text { KLD } & \text { Kullback Leibler distance } \\ \text { MB } & \text { Maxwell-Boltzmann } \\ \text { MI } & \text { Mutual information } \\ \text { MMA } & \text { Multi modulus algorithm } \\ \text { NLPN } & \text { Nonlinear phase noise } \\ \text { NLSE } & \text { Nonlinear Schrödinger equation } \\ \text { OPC } & \text { Optical phase conjugation } \\ \text { PDF } & \text { Probability density function } \\ \text { PMF } & \text { Probability mass function } \\ \text { PN } & \text { Phase noise } \\ \text { PS } & \text { Probabilistic shaping } \\ \text { QAM } & \text { Quadrature amplitude modulation } \\ \text { SNR } & \text { Signal to noise ratio } \\ \text { SSFM } & \text { Split step Fourier method } \\ \text { SSMF } & \text { Standard single mode fiber } \\ \text { WDM } & \text { Wavelength division multiplexing } \\ \text { ZC } & \text { Zadoff-Chu } \\ & \end{array}$




\section{ACKNOWLEDGMENT}

This work was supported by the DNRF Research Centre of Excellence, SPOC, ref. DNRF123. The work of Francesco Da Ros is supported by the Villum Foundation through the Villum Young Investigator fellowship OPTIC-AI (grant no. 29344). The work of Edson Porto da Silva is supported by the National Council for Scientific and Technological Development (CNPq), Brazil, under the Grant 432214/2018-6. OFS Denmark is acknowledged for providing the highly-nonlinear fiber used in this work.

\section{REFERENCES}

[1] G. Kramer, M. I. Yousefi, and F. R. Kschischang, "Upper bound on the capacity of a cascade of nonlinear and noisy channels," in 2015 IEEE Information Theory Workshop (ITW), 2015, pp. 1-4.

[2] M. Secondini, E. Agrell, E. Forestieri, D. Marsella, and M. R. Camara, "Nonlinearity mitigation in WDM systems: Models, strategies, and achievable rates," IEEE/OSA Journal of Lightwave Technology, vol. 37, no. 10 , pp. 2270-2283, 2019

[3] J. C. Cartledge, F. P. Guiomar, F. R. Kschischang, G. Liga, and M. P. Yankov, "Digital signal processing for fiber nonlinearities [invited]," Optics Express, vol. 25, no. 3, pp. 1916-1936, Feb 2017.

[4] R. Essiambre, G. Kramer, P. J. Winzer, G. J. Foschini, and B. Goebel, "Capacity limits of optical fiber networks," IEEE/OSA Journal of Lightwave Technology, vol. 28, no. 4, pp. 662-701, 2010.

[5] A. Ellis, M. McCarthy, M. Al Khateeb, M. Sorokina, and N. Doran, "Performance limits in optical communications due to fiber nonlinearity," Advances in Optics and Photonics, vol. 9, no. 3, pp. 429-503, 2017.

[6] F. Da Ros, M. Lilleholm, M. P. Yankov, P. Guan, H. Hu, S. Forchhammer, M. Galili, and L. K. Oxenløwe, "Impact of signal-conjugate wavelength shift on optical phase conjugation-based transmission of QAM signals," in Proc. of European Conference on Optical Communications, ECOC, Sep. 2017, p. P1.SC4.66.

[7] S. T. Le, V. Aref, and H. Buelow, "Nonlinear signal multiplexing for communication beyond the Kerr nonlinearity limit," Nature Photonics, vol. 11 , no. 9, p. 570, 2017.

[8] F. P. Guiomar and A. N. Pinto, "Simplified Volterra series nonlinear equalizer for polarization-multiplexed coherent optical systems," IEEE/OSA Journal of Lightwave Technology, vol. 31, no. 23, pp. 38793891, Dec 2013

[9] E. P. da Silva, M. P. Yankov, F. D. Ros, T. Morioka, and L. K. Oxenløwe, "Perturbation-based FEC-assisted iterative nonlinearity compensation for wdm systems," IEEE/OSA Journal of Lightwave Technology, vol. 37, no. 3, pp. 875-881, Feb 2019.

[10] G. Liga, T. Xu, A. Alvarado, R. I. Killey, and P. Bayvel, "On the performance of multichannel digital backpropagation in high-capacity long-haul optical transmission," Optics Express, vol. 22, no. 24, pp. 30 053-30 062, Dec 2014

[11] R. Dar and P. J. Winzer, "Nonlinear interference mitigation: Methods and potential gain," Journal of Lightwave Technology, vol. 35, no. 4, pp. 903-930, 2017.

[12] R. F. Fischer, Precoding and Signal Shaping for Digital Transmission. USA: John Wiley \& Sons, Inc., 2002.

[13] G. D. Forney, "Trellis shaping," IEEE Transactions on Information Theory, vol. 38, no. 2, pp. 281-300, 1992.

[14] A. K. Khandani and P. Kabal, "Shaping multidimensional signal spaces. I. optimum shaping, shell mapping," IEEE Transactions on Information Theory, vol. 39, no. 6, pp. 1799-1808, 1993.

[15] C. E. Shannon, "A mathematical theory of communication," The Bell System Technical Journal, vol. 27, pp. 379-423, 1948.

[16] G. Böcherer, F. Steiner, and P. Schulte, "Bandwidth efficient and ratematched low-density parity-check coded modulation," IEEE Transactions on Communications, vol. 63, no. 12, pp. 4651-4665, 2015.

[17] D. Raphaeli and A. Gurevitz, "Constellation shaping for pragmatic turbo-coded modulation with high spectral efficiency," IEEE Transactions on Communications, vol. 52, no. 3, pp. 341-345, 2004.

[18] M. Yankov, S. Forchhammer, K. J. Larsen, and L. P. B. Christensen, "Rate-adaptive constellation shaping for near-capacity achieving turbo coded BICM," in 2014 IEEE International Conference on Communications (ICC), 2014, pp. 2112-2117.
[19] M. C. Valenti and X. Xiang, "Constellation shaping for bit-interleaved LDPC coded APSK," IEEE Transactions on Communications, vol. 60 , no. 10, pp. 2960-2970, 2012.

[20] L. Beygi, E. Agrell, J. M. Kahn, and M. Karlsson, "Rate-adaptive coded modulation for fiber-optic communications," IEEE/OSA Journal of Lightwave Technology, vol. 32, no. 2, pp. 333-343, 2014.

[21] B. P. Smith and F. R. Kschischang, "A pragmatic coded modulation scheme for high-spectral-efficiency fiber-optic communications," IEEE/OSA Journal of Lightwave Technology, vol. 30, no. 13, pp. 20472053, 2012.

[22] M. P. Yankov, F. Da Ros, E. P. da Silva, S. Forchhammer, K. J. Larsen, L. K. Oxenløwe, M. Galili, and D. Zibar, "Constellation shaping for WDM systems using 256QAM/1024QAM with probabilistic optimization," IEEE/OSA Journal of Lightwave Technology, vol. 34, no. 22, pp. 5146-5156, Nov. 2016.

[23] F. Buchali, F. Steiner, G. Böcherer, L. Schmalen, P. Schulte, and W. Idler, "Rate adaptation and reach increase by probabilistically shaped 64-QAM: An experimental demonstration," IEEE/OSA Journal of Lightwave Technology, vol. 34, no. 7, pp. 1599-1609, 2016.

[24] N. Merhav, G. Kaplan, A. Lapidoth, and S. S. Shitz, "A generalization of the Blahut-Arimoto algorithm to finite-state channels," IEEE Transactions on Information Theory, vol. 40, no. 6, pp. 1953-1967, 1994.

[25] I. Csiszar and J. Korner, "Graph decomposition: A new key to coding theorems," IEEE Transactions on Information Theory, vol. 27, no. 1, pp. 5-12, 1981.

[26] M. P. Yankov, F. D. Ros, E. P. da Silva, S. Forchhammer, M. Galili, and L. K. Oxenløwe, "Optimizing the achievable rates of tricky channels: A probabilistic shaping for OPC channel example," in 2018 IEEE Photonics Conference (IPC), 2018, pp. 1-2.

[27] M. P. Yankov, K. J. Larsen, and S. Forchhammer, "Temporal probabilistic shaping for mitigation of nonlinearities in optical fiber systems," IEEE/OSA Journal of Lightwave Technology, vol. 35, no. 10, pp. 1803 1810, 2017.

[28] J. Renner, T. Fehenberger, M. P. Yankov, F. Da Ros, S. Forchhammer, G. Böcherer, and N. Hanik, "Experimental comparison of probabilistic shaping methods for unrepeated fiber transmission," IEEE/OSA Journal of Lightwave Technology, vol. 35, no. 22, pp. 4871-4879, 2017.

[29] P. Schulte, F. Steiner, and G. Böcherer, "Four dimensional probabilistic shaping for fiber-optic communication," in Advanced Photonics 2017 (IPR, NOMA, Sensors, Networks, SPPCom, PS), 2017, p. SpM2F.5.

[30] A. D. Ellis et al., "4 Tb/s transmission reach enhancement using 10x400 $\mathrm{Gb} / \mathrm{s}$ super-channels and polarization insensitive dual band optical phase conjugation," IEEE/OSA Journal of Lightwave Technology, vol. 34, no. 8, pp. 1717-1723, Apr. 2016.

[31] P. Kaminski, F. D. Ros, A. T. Clausen, S. Forchhammer, L. Oxenløwe, and M. Galili, "Improved nonlinearity compensation of opc-aided edfaamplified transmission by enhanced dispersion mapping," in Conference on Lasers and Electro-Optics. Optical Society of America, 2020, p. STu3L.4.

[32] P. Minzioni, "Nonlinearity compensation in a fiber-optic link by optical phase conjugation," Fiber and Integrated Optics, vol. 28, no. 3, pp. 179-209, 2009

[33] C. Sánchez, M. McCarthy, A. Ellis, P. Wright, and A. Lord, "Opticalphase conjugation nonlinearity compensation in flexi-grid optical networks," in Recent advances on systems, signals, control, communications and computers, dec 2015, pp. 39-43.

[34] K. Solis-Trapala, M. Pelusi, H. N. Tan, T. Inoue, and S. Namiki, "Optimized WDM transmission impairment mitigation by multiple phase conjugations," Journal of Lightwave Technology, vol. 34, no. 2, pp. 431440, 2015.

[35] H. Hu, R. M. Jopson, A. H. Gnauck, S. Randel, and S. Chandrasekhar, "Fiber nonlinearity mitigation of WDM-PDM QPSK/16-QAM signals using fiber-optic parametric amplifiers based multiple optical phase conjugations," Optics express, vol. 25, no. 3, pp. 1618-1628, 2017.

[36] I. Sackey, F. Da Ros, M. Jazayerifar, T. Richter, C. Meuer, M. Nölle, L. Molle, C. Peucheret, K. Petermann, and C. Schubert, "Kerr nonlinearity mitigation in $5 \times 28$-GBd PDM 16-QAM signal transmission over a dispersion-uncompensated link with backward-pumped distributed Raman amplification," Optics Express, vol. 22, no. 22, pp. 27381 27 391, 2014.

[37] M. Tan, M. Al-Khateeb, T. Zhang, P. Harper, and A. D. Ellis, "Distributed Raman amplification design for fibre nonlinearity compensation with mid-link optical phase conjugation," in 2019 21st International Conference on Transparent Optical Networks (ICTON). IEEE, 2019, pp. $1-4$.

[38] J. C. Cartledge, A. D. Ellis, A. Shiner, A. A. El-Rahman, M. McCarthy, M. Reimer, A. Borowiec, and A. Kashi, "Signal processing techniques 
for reducing the impact of fiber nonlinearities on system performance," in Optical Fiber Communication Conference. Optical Society of America, 2016, pp. Th4F-5.

[39] G. Liga, G. Saavedra, and P. Bayvel, "Combining optical phase conjugation and Volterra equalisation: A novel nonlinearity compensation scheme," in 2018 European Conference on Optical Communication (ECOC). IEEE, 2018, pp. 1-3.

[40] G. Saavedra, G. Liga, and P. Bayvel, "Volterra-assisted optical phase conjugation: A hybrid optical-digital scheme for fiber nonlinearity compensation," IEEE/OSA Journal of Lightwave Technology, vol. 37, no. 10, pp. 2467-2479, 2019

[41] H. E. Hansen, M. P. Yankov, P. M. Kaminski, F. D. Ros, L. K. Oxenløwe, and S. Forchhammer, "Noise statistics and its implications on optimal constellation shapes for channels with optical phase conjugation," in Conference on Lasers and Electro-Optics. Optical Society of America, 2020, p. STu3L.5.

[42] D. M. Arnold, H.-A. Loeliger, P. O. Vontobel, A. Kavčić, and W. Zeng, "Simulation-based computation of information rates for channels with memory," IEEE Transactions on Information Theory, vol. 52, no. 8, pp. 3498-3508, Aug. 2006.

[43] O. E. Agazzi, M. R. Hueda, H. S. Carrer, and D. E. Crivelli, "Maximum-likelihood sequence estimation in dispersive optical channels," IEEE/OSA Journal of Lightwave Technology, vol. 23, no. 2, pp. 749-763, 2005.

[44] G. Bosco, P. Poggiolini, and M. Visintin, "Performance analysis of MLSE receivers based on the square-root metric," IEEE/OSA Journal of Lightwave Technology, vol. 26, no. 14, pp. 2098-2109, 2008.

[45] D. Marsella, M. Secondini, and E. Forestieri, "Maximum likelihood sequence detection for mitigating nonlinear effects," IEEE/OSA Journal of Lightwave Technology, vol. 32, no. 5, pp. 908-916, 2014.

[46] G. Liga, A. Alvarado, E. Agrell, M. Secondini, R. I. Killey, and P. Bayvel, "Optimum detection in presence of nonlinear distortions with memory," in 2019 European Conference on Optical Communication (ECOC), 2015, pp. 1-3.

[47] R. T. Jones, M. P. Yankov, and D. Zibar, "End-to-end learning for GMI optimized geometric constellation shape," in 2019 European Conference on Optical Communication (ECOC), 2020, pp. 1-3.

[48] T. A. Eriksson, T. Fehenberger, P. A. Andrekson, M. Karlsson, N. Hanik, and E. Agrell, "Impact of 4D channel distribution on the achievable rates in coherent optical communication experiments," IEEE/OSA Journal of Lightwave Technology, vol. 34, no. 9, pp. 2256-2266, 2016.

[49] Y. Wu and S. Verdú, "The impact of constellation cardinality on Gaussian channel capacity," in 2010 48th Annual Allerton Conference on Communication, Control, and Computing (Allerton), 2010, pp. 620-628.

[50] F. R. Kschischang and S. Pasupathy, "Optimal nonuniform signaling for Gaussian channels," IEEE Transactions on Information Theory, vol. 39 , no. 3, pp. 913-929, 1993.

[51] N. Varnica, X. Ma, and A. Kavčić, "Capacity of power constrained memoryless AWGN channels with fixed input constellations," in Proc. of IEEE Globecom, Nov. 2002, pp. 1339-1343.

[52] J. Bellorado, S. Ghassemzadeh, and A. Kavcic, "Approaching the capacity of the MIMO rayleigh flat-fading channel with qam constellations, independent across antennas and dimensions," IEEE Transactions on Wireless Communications, vol. 5, no. 6, pp. 1322-1332, 2006.

[53] T. M. Cover and J. A. Thomas, Elements of Information Theory, 2nd edition. USA: Wiley-Interscience, 2006.

[54] P. O. Vontobel, A. Kavcic, D. M. Arnold, and H. Loeliger, "A generalization of the Blahut-Arimoto algorithm to finite-state channels," IEEE Transactions on Information Theory, vol. 54, no. 5, pp. 1887-1918, 2008.

[55] S. J. Savory, "Digital filters for coherent optical receivers," Optics Express, vol. 16, no. 2, pp. 804-817, Jan. 2008.

[56] F. Da Ros, M. P. Yankov, E. P. da Silva, M. Lillieholm, S. Forchhammer, M. Galili, L. K. Oxenløwe et al., "Link-placement characterization of optical phase conjugation for nonlinearity compensation," in $2018 \mathrm{Op}$ tical Fiber Communications Conference and Exposition (OFC). IEEE, 2018, pp. 1-3.

[57] A. Ellis, M. McCarthy, M. Al-Khateeb, and S. Sygletos, "Capacity limits of systems employing multiple optical phase conjugators," Optics express, vol. 23, no. 16, pp. 20381-20393, 2015

[58] H. Hu, M. P. Yankov, F. Da Ros, Y. Amma, Y. Sasaki, T. Mizuno, Y. Miyamoto, M. Galili, S. Forchhammer, L. K. Oxenløwe, and T. Morioka, "Ultrahigh-spectral-efficiency WDM/SDM transmission using PDM-1024-QAM probabilistic shaping with adaptive rate," IEEE/OSA Journal of Lightwave Technology, vol. 36, no. 6, pp. 1304 1308,2018
[59] M. P. Yankov, E. P. da Silva, F. Da Ros, and D. Zibar, "Experimental analysis of pilot-based equalization for probabilistically shaped WDM systems with 256QAM/1024QAM," in 2017 Optical Fiber Communications Conference and Exhibition (OFC), 2017, pp. 1-3.

[60] M. P. Yankov, T. Fehenberger, L. Barletta, and N. Hanik, "Lowcomplexity tracking of laser and nonlinear phase noise in wdm optical fiber systems," IEEE/OSA Journal of Lightwave Technology, vol. 33 , no. 23, pp. 4975-4984, 2015.

[61] M. P. Yankov, F. Da Ros, E. P. da Silva, T. Fehenberger, L. Barletta, D. Zibar, L. K. Oxenløwe, M. Galili, and S. Forchhammer, "Nonlinear phase noise compensation in experimental wdm systems with 256QAM," IEEE/OSA Journal of Lightwave Technology, vol. 35, no. 8, pp. 1438$1443,2017$.

[62] M. P. Yankov, L. Barletta, and D. Zibar, "Phase noise compensation for nonlinearity-tolerant digital subcarrier systems with high-order QAM," IEEE Photonics Journal, vol. 9, no. 5, pp. 1-12, 2017.

[63] D. S. Millar, R. Maher, D. Lavery, T. Koike-Akino, M. Pajovic, A. Alvarado, M. Paskov, K. Kojima, K. Parsons, B. C. Thomsen, S. J. Savory, and P. Bayvel, "Design of a $1 \mathrm{~Tb} / \mathrm{s}$ superchannel coherent receiver," IEEE/OSA Journal of Lightwave Technology, vol. 34, no. 6, pp. 14531463, 2016.

[64] M. Pajovic, D. S. Millar, T. Koike-Akino, R. Maher, D. Lavery, A. Alvarado, M. Paskov, K. Kojima, K. Parsons, B. C. Thomsen, S. J. Savory, and P. Bayvel, "Experimental demonstration of multi-pilot aided carrier phase estimation for DP-64QAM and DP-256QAM," in 2015 European Conference on Optical Communication (ECOC), 2015, pp. 1-3.

[65] 3GPP.TS.36.211, "Evolved universal terrestrial radio access (E-UTRA); physical channels and modulation." 3rd Generation Partnership Project, Technical Specification Group Radio Access Network.

[66] I. Fatadin, D. Ives, and S. J. Savory, "Blind equalization and carrier phase recovery in a 16-QAM optical coherent system," IEEE/OSA Journal of Lightwave Technology, vol. 27, no. 15, pp. 3042-3049, 2009.

[67] F. A. Barbosa, S. M. Rossi, and D. A. A. Mello, "Phase and frequency recovery algorithms for probabilistically shaped transmission," IEEE/OSA Journal of Lightwave Technology, vol. 38, no. 7, pp. 1827-1835, 2020.

[68] D. Godard, "Self-recovering equalization and carrier tracking in twodimensional data communication systems," IEEE Transactions on Communications, vol. 28, no. 11, pp. 1867-1875, 1980.

[69] S. Dris, S. Alreesh, and A. Richter, "Blind polarization demultiplexing and equalization of probabilistically shaped QAM," in 2019 Optical Fiber Communications Conference and Exhibition (OFC), 2019, pp. 13.

[70] M. Sorokina, S. Sygletos, and S. Turitsyn, "Ripple distribution for nonlinear fiber-optic channels," Optics Express, vol. 25, no. 3, pp. 2228 2238, Feb. 2017.

[71] T. Freckmann, R. J. Essiambre, P. J. Winzer, G. J. Foschini, and G. Kramer, "Fiber capacity limits with optimized ring constellations," IEEE Photonics Technology Letters, vol. 21, no. 20, pp. 1496-1498, 2009.

[72] E. Porto da Silva, M. Yankov, F. Da Ros, S. Forchhammer, M. Galili, L. Oxenløwe, and D. Zibar, "Experimental comparison of gains in achievable information rates from probabilistic shaping and digital backpropagation for DP-256QAM/1024QAM WDM systems," in Proceedings of the European Conference on Optical Communication, 2016.

[73] O. V. Sinkin, R. Holzlohner, J. Zweck, and C. R. Menyuk, "Optimization of the split-step Fourier method in modeling optical-fiber communications systems," IEEE/OSA Journal of Lightwave Technology, vol. 21, no. 1, pp. 61-68, 2003.

[74] F. D. Ros, E. da Silva, A. Gajda, P. Kaminski, V. Cristofori, A. Peczek, A. Mai, K. Petermann, L. Zimmermann, L. Oxenløwe, and M. Galili, "Nonlinearity compensation for dual-polarization signals using optical phase conjugation in a silicon waveguide," in Conference on Lasers and Electro-Optics. Optical Society of America, 2018, p. STu4C.1.

[75] L. B. Du and A. J. Lowery, "The validity of "Odd and Even" channels for testing all-optical OFDM and Nyquist WDM long-haul fiber systems," Optics Express, vol. 20, no. 26, pp. B445-B451, Dec 2012.

[76] R. Dar, S. Chandrasekhar, A. H. Gnauck, B. Li, J. Cho, E. C. Burrows, and P. J. Winzer, "Impact of WDM channel correlations on nonlinear transmission," in ECOC 2016; 42nd European Conference on Optical Communication, 2016, pp. 1-3.

[77] R. Dar, M. Feder, A. Mecozzi, and M. Shtaif, "On shaping gain in the nonlinear fiber-optic channel," in 2014 IEEE International Symposium on Information Theory, 2014, pp. 2794-2798.

[78] M. Reimer, S. O. Gharan, A. D. Shiner, and M. O'Sullivan, "Optimized 4 and 8 dimensional modulation formats for variable capacity in optical networks," in 2016 Optical Fiber Communications Conference and Exhibition (OFC), 2016, pp. 1-3. 
[79] O. Geller, R. Dar, M. Feder, and M. Shtaif, "A shaping algorithm for mitigating inter-channel nonlinear phase-noise in nonlinear fiber systems," IEEE/OSA Journal of Lightwave Technology, vol. 34, no. 16, pp. 3884-3889, 2016

Metodi Plamenov Yankov (M'13) received a B. Eng. degree from the Technical University of Sofia, Bulgaria in 2010 in the field of radio communications, a M. Sc. degree from the Technical University of Denmark (DTU), Lyngby in 2012 in the area of signals and transmission technology for telecommunications and a $\mathrm{PhD}$ degree from the Technical University of Denmark on the topic of constellation constrained capacity estimation nearcapacity achieving digital methods. He held a post-doc position at the DNRF Centre of Excellence SPOC (2015-2017) and an industrial post-doc position at Fingerprint Cards A/S, Denmark (2017-2019). Since, he has been employed at DTU Fotonik, the Coding and Visual Communications group as a researcher His research interests include among others information and communication theory of digital transceivers, digital signal processing techniques in general and for wireless and optical fiber communications in particular, machine learning, forward error correction codes, and information theory of biometrics. $\mathrm{He}$ is currently a member of the IEEE Photonics society.

Henrik Enggaard Hansen received a master of science in telecommunication engineering at the Technical University of Denmark in 2018. He is currently enrolled as a Ph.D. student at DTU Fotonik, Department of Photonics at his alma mater, where he is a part of the Centre of Excellence in Silicon Photonics for Optical Communication. His research interests include signal processing for communication systems, high spectral efficiency communication and signal processing for nonlinear fiber optics.

Francesco Da Ros received his B.Sc. degree in information engineering from Università degli Studi di Padova, Italy, in 2008. He also received a dual M.Sc. degree in telecommunication engineering from Università degli Studi di Padova, Italy, and Technical University of Denmark (DTU), Denmark, in 2011 and the Ph.D. degree from DTU in 2014. Between 2013 and 2014 he spent a seven-month research stay at Fraunhofer Institute for Telecommunications, Heinrich-Hertz-Institute, Germany. Between 2015 and 2018, he has been working as a post-doc researcher at DTU within the Center for Silicon Photonics for Optical Communications (SPOC) focusing on all-optical signal processing and nonlinearity compensation techniques for optical transmission systems. Since 2019, he is a researcher in the Machine Learning in Photonic Systems group at DTU Fotonik, focusing on the nonlinear Fourier transform and machine learning techniques applied to coherent communications. Dr. Da Ros has co-authored more than 100 journal and conference papers. He is an OSA Young Professional Volunteer member, an IEEE Young Professional member and has been serving as a technical subcommittee member of the Conference on Lasers and Electro Optics (CLEO) since 2017, chairing a subcommittee it since 2019 .

Pawel Marcin Kaminski received his B.Sc. degree in telecommunications and computer science from Lodz University of Technology, Poland, in 2015. He then received the M.Sc. degree in photonics engineering from the Technical University of Denmark (DTU), Denmark, in 2017, where he is now working towards his Ph.D. degree in high-speed optical communications. He is currently researching optical signal processing techniques for the Kerr nonlinearity suppression in optical systems. Between 2017 and 2020, he visited the National Institute of Information and Communications (NICT), Japan, and the Research and Development Centre in Telecommunications (CPQD), Brazil, where he conducted research on novel optical phase conjugation-aided systems. He has authored and co-authored more than 12 conference or journal publications on optical signal processing and nonlinear transmission.
Edson P. da Silva (SM'19) was born in Pocinhos, Brazil, on August 9, 1988. He received a master's degree in electrical engineering from the State University of Campinas (UNICAMP), Campinas, Brazil, in 2013, and the Ph.D. degree in photonics engineering from the Technical University of Denmark (DTU Fotonik), Kongens Lyngby, Denmark, in 2017. Between June and October 2016, he was a visiting researcher with the Telecommunications Department, Technical University of Munich (TUM), Germany. Between April 2017 and July 2018, he was a Postdoctoral with the Silicon Photonics for Optical Communication (SPOC) Center of Excellence, DTU Fotonik. Since August 2018, he has been an Adjunct Professor with the Electrical Engineering Department, Federal University of Campina Grande (UFCG), Campina Grande, Brazil. His research topics involve digital signal processing for coherent optical transceivers, mitigation of non-linear distortions in optical channels, and high-speed coherent optical transmission. Together with other members of DTU's high-speed optical communications research group, researchers from Southampton University, and Fujikura Ltd., he has received from the European Commission the Horizon Prize, in 2016, for contributions made to the advancement of optical transmission technologies

Michael Galili (Aabenraa, Denmark, 1977) obtained his MSc in Engineering in applied physics, from the Technical University of Denmark (DTU), in 2003 and his Ph.D. in Optical communications and signal processing from DTU Fotonik in 2007. He is currently Associate Professor and scientific coordinator in the research center Silicon Photonics for Optical Communication (SPOC). $\mathrm{He}$ is the first author or co-author of more than 300 peer reviewed journal and conference publications and he is teaching and supervising students at bachelor, master and $\mathrm{PhD}$ level. His research interests include optical transmission and signal processing for telecommunication, nonlinear integrated devices for optical processing and advanced optical switches for Datacom systems.

Leif Katsuo Oxenløwe is the group leader of the High-Speed Optical Communications group at DTU Fotonik, Department of Photonics Engineering, at the Technical University of Denmark (DTU), and the Centre Leader of the Research Centre of Excellence SPOC (Silicon Photonics for Optical Communications) supported by the Danish National Research Foundation. He is the recipient of a Top-Researcher grant from the Danish Research Council (DFF) for the project NANO-SPECs. He is also the recipient of a European Research Council grant (project SOCRATES) focusing on the use of ultra-high-speed serial data for Ethernet networks, and he is involved in several other national and international projects exploring nonlinear optics in fibres and devices for optical signal processing. He has authored or co-authored more than 350 peer reviewed publications, including 15 postdeadline papers at major conferences, 5 book chapters and he holds 7 patents. In 2009 he was awarded the Elektropris from Elektrofondet, and he has received 4 best paper awards. He received the B.Sc. degree in physics and astronomy from the Niels Bohr Institute, University of Copenhagen, Denmark in 1996. In 1998 he received the International Diploma of Imperial College, London, UK and the M.Sc. degree from the University of Copenhagen. He received the Ph.D. degree in 2002 from DTU and is since 2009 Professor of Photonic Communication Technologies. In 2016, SPOC won The Horizon Prize - Breaking the optical transmission barriers with the proposal PHOTONMAP (PHOtonic chip enabled large-capacity data TransmissiON with high- count Multi-core fibres and AmPlifiers) https://ec.europa.eu/research/horizonprize/index.cfm?prize=opticaltransmission

Søren Forchhammer (M'04) received the M.S. degree in engineering and the Ph.D. degree from the Technical University of Denmark, Lyngby, in 1984 and 1988, respectively. Currently, he is a Professor with DTU Fotonik, Technical University of Denmark, where he has been since 1988. He is Head of the Coding and Visual Communication Group at DTU Fotonik. His main interests include, signal and image processing, source coding, distributed coding, visual communication technology, information theory and signal processing and coding for optical communication. 